\title{
Observational calibration of the projection factor of Cepheids
}

\section{The long-period Galactic Cepheid RS Puppis ${ }^{\star}$}

\author{
Pierre Kervella ${ }^{1,2}$, Boris Trahin ${ }^{1,2}$, Howard E. Bond ${ }^{3}$, Alexandre Gallenne ${ }^{4}$, Laszlo Szabados ${ }^{5}$, Antoine Mérand ${ }^{4}$, \\ Joanne Breitfelder ${ }^{2,4}$, Julien Dailloux ${ }^{1,6}$, Richard I. Anderson ${ }^{7,8}$, Pascal Fouqué ${ }^{9,10}$, Wolfgang Gieren ${ }^{11}$, \\ Nicolas Nardetto ${ }^{12}$, and Grzegorz Pietrzyński ${ }^{13}$ \\ ${ }^{1}$ Unidad Mixta Internacional Franco-Chilena de Astronomía (CNRS UMI 3386), Departamento de Astronomía, \\ Universidad de Chile, Camino El Observatorio 1515, Las Condes, Santiago, Chile \\ e-mail: pkervell@das.uchile.cl \\ 2 LESIA (UMR 8109), Observatoire de Paris, PSL Research University, CNRS, UPMC, Univ. Paris-Diderot, 5 place Jules Janssen, \\ 92195 Meudon, France \\ e-mail: pierre.kervella@obspm.fr \\ 3 Department of Astronomy \& Astrophysics, 525 Davey Lab., Pennsylvania State University, University Park, PA 16802 USA \\ 4 European Southern Observatory, 3107 Alonso de Córdova, Casilla 19001, Santiago 19, Chile \\ 5 Konkoly Observatory, MTA CSFK, Konkoly Thege M. út 15-17, 1121 Budapest Hungary \\ 6 Institut Supérieur de l'Aéronautique et de l'Espace, 10 avenue Édouard Belin, 31400 Toulouse, France \\ 7 Physics and Astronomy Department, The Johns Hopkins University, 3400 N. Charles St., Baltimore, MD 21218, USA \\ 8 Observatoire de Genève, Université de Genève, 51 Ch. des Maillettes, 1290 Sauverny, Switzerland \\ 9 IRAP, UMR 5277, CNRS, Université de Toulouse, 14 avenue Édouard Belin, 31400 Toulouse, France \\ 10 CFHT Corporation, 65-1238 Mamalahoa Hwy, Kamuela, Hawaii 96743, USA \\ 11 Universidad de Concepción, Departamento de Astronomía, Casilla 160-C, Concepción, Chile \\ 12 Laboratoire Lagrange, UMR7293, Université de Nice Sophia-Antipolis, CNRS, Observatoire de la Côte d'Azur, 06000 Nice, \\ France \\ 13 Nicolaus Copernicus Astronomical Center, Polish Academy of Sciences, ul. Bartycka 18, 00-716 Warszawa, Poland
}

Received 6 December 2016 / Accepted 11 January 2017

\begin{abstract}
The projection factor ( $p$-factor) is an essential component of the classical Baade-Wesselink (BW) technique, which is commonly used to determine the distances to pulsating stars. It is a multiplicative parameter used to convert radial velocities into pulsational velocities. As the BW distances are linearly proportional to the $p$-factor, its accurate calibration for Cepheids is of critical importance for the reliability of their distance scale. We focus on the observational determination of the $p$-factor of the long-period Cepheid RS Pup $(P=41.5$ days). This star is particularly important as this is one of the brightest Cepheids in the Galaxy and an analog of the Cepheids used to determine extragalactic distances. An accurate distance of $1910 \pm 80 \mathrm{pc}( \pm 4.2 \%)$ has recently been determined for RS Pup using the light echoes propagating in its circumstellar nebula. We combine this distance with new VLTI/PIONIER interferometric angular diameters, photometry, and radial velocities to derive the $p$-factor of RS Pup using the code Spectro-Photo-Interferometry of Pulsating Stars (SPIPS). We obtain $p=1.250 \pm 0.064( \pm 5.1 \%)$, defined for cross-correlation radial velocities. Together with measurements from the literature, the $p$-factor of RS Pup confirms the good agreement of a constant $\bar{p}=1.293 \pm 0.039( \pm 3.0 \%)$ model with the observations. We conclude that the $p$-factor of Cepheids is constant or mildly variable over a broad range of periods (3.7 to 41.5 days).
\end{abstract}

Key words. stars: individual: RS Pup - stars: variables: Cepheids - techniques: interferometric - techniques: photometric stars: distances - distance scale

\section{Introduction}

The oscillation period of Cepheids is longer for more massive, less dense, and more luminous stars. This cyclic change in radius, and its associated effective temperature modulation,

\footnotetext{
* Based on observations collected at the European Organisation for Astronomical Research in the Southern Hemisphere under ESO programs 093.D-0316(A), 094.D-0773(B), 096.D-0341(A) and 098.D0067(A). Based in part on observations with the $1.3 \mathrm{~m}$ telescope operated by the SMARTS Consortium at Cerro Tololo Interamerican Observatory.
}

is the physical basis of the empirical Leavitt law (the PeriodLuminosity relation, Leavitt 1908; Leavitt \& Pickering 1912). The calibration of the zero-point of the Leavitt law requires the independent measurement of the distances of a sample of Cepheids. This is complicated by the rarity of these massive stars, and particularly the long-period oscillators, which results in large distances beyond the capabilities of trigonometric parallax measurements. The parallax-of-pulsation method, also known as the Baade-Wesselink (BW) technique, is a powerful technique to measure the distances to individual Galactic and LMC Cepheids. The variation of the angular diameter of the star 
A\&A 600, A127 (2017)

Table 1. Characteristics of the calibrators used for the PIONIER observations of RS Pup.

\begin{tabular}{clllllllc}
\hline \hline Number & Name & Sp.type & $m_{B}$ & $m_{V}$ & $m_{H}$ & $m_{K}$ & $\begin{array}{c}\theta_{\mathrm{LD}} \\
(\mathrm{mas})\end{array}$ & $\begin{array}{c}\theta_{\mathrm{UD} \mathrm{H}} \\
(\mathrm{mas})\end{array}$ \\
\hline 1 & HD 67977 & G8III & 7.10 & 6.21 & 4.29 & 4.06 & $0.738 \pm 0.020$ & $0.713 \pm 0.020$ \\
2 & HD 69002 & K2III & 7.54 & 6.37 & 3.99 & 3.84 & $0.867 \pm 0.020$ & $0.838 \pm 0.020$ \\
3 & HD 68978 & G2V & 7.33 & 6.71 & 5.37 & 5.27 & $0.374 \pm 0.008$ & $0.361 \pm 0.008$ \\
4 & HD 73947 & K2III & 8.48 & 7.09 & 3.95 & 3.88 & $0.863 \pm 0.012$ & $0.834 \pm 0.012$ \\
\hline
\end{tabular}

Notes. For calibrators 1 to 3, we employed the surface brightness color relations calibrated by Kervella et al. (2004) to estimate their angular diameters. They were selected from the catalogs of Lafrasse et al. (2010a,b) (1 to 3) and Mérand et al. (2005a) (4).

(from surface brightness-color relations or optical interferometry) is compared to the variation of the linear diameter (from the integration of the radial velocity). The distance of the Cepheid is then obtained by simultaneously fitting the linear and angular amplitudes (see, e.g., Storm et al. 2011). The main weakness of the BW technique is that it uses a numerical factor to convert disk-integrated radial velocities into photospheric velocities, the projection factor, or $p$-factor (Nardetto et al. 2007; Barnes 2009; Nardetto et al. 2014b). This factor, whose expected value is typically around 1.3 , simultaneously characterizes the spherical geometry of the pulsating star, the limb darkening, and the difference in velocity between the photosphere and the line-forming regions. Owing to this intrinsic complexity, the $p$-factor is currently uncertain to $5-10 \%$, and accounts for almost all the systematic uncertainties of the nearby Cepheid BW distances. This is the main reason why Galactic Cepheids were excluded from the measurement of $H_{0}$ by Riess et al. (2011).

Recent observational efforts have produced accurate measurements of the $p$-factor of Cepheids (Mérand et al. 2005b; Pilecki et al. 2013; Breitfelder et al. 2015, 2016; Gieren et al. 2015), with the objective to reduce this source of systematic uncertainty. However, these $p$-factor calibrations up to now were essentially obtained on low-luminosity, relatively short-period Cepheids ( $P \lesssim 10$ days) that are the most common in the Galaxy. The most important Cepheids for extragalactic distance determinations are the long-period pulsators ( $P \gtrsim 10$ days), however. A calibration of the $p$-factor of the intrinsically brightest Cepheids is therefore highly desirable. Theoretical studies (e.g., Neilson et al. 2012) indicate that the $p$-factor may vary with the period, but the dependence differs between authors (Nardetto et al. 2014a; Storm et al. 2011; Breitfelder et al. 2016).

We focus the present study on the long-period Cepheid RS Pup (HD 68860, HIP 40233, SAO 198944). Its period of $P=41.5$ days makes it one of the brightest Cepheids of our Galaxy and the second nearest long-period pulsator after $\ell$ Carinae (HD 84810, $P=35.55$ days). Kervella et al. (2014) reported an accurate measurement of the distance of RS Pup, $d=$ $1910 \pm 80 \mathrm{pc}$, corresponding to a parallax $\pi=0.524 \pm 0.022$ mas. This distance was obtained from a combination of photometry and polarimetry of the light echoes that propagate in its circumstellar dust nebula. It is in agreement with the GaiaTGAS parallax of $\pi=0.63 \pm 0.26$ mas (Gaia Collaboration et al. 2016a), whose systematic uncertainty is estimated to \pm 0.3 mas by Lindegren et al. (2016). In the present work, we employ the light echo distance of RS Pup, in conjunction with new interferometric angular diameter measurements, photometry, and archival data (Sect. 2) to apply the Spectro-Photo-Interferometry of Pulsating Stars (SPIPS) modeling (Sect. 3). Through this inverse version of the parallax-of-pulsation technique, we derive its $p$ factor and compare it to the values obtained for $\ell$ Car and other Cepheids (Sect. 4).

\section{Observations}

\subsection{Interferometry}

We observed RSPup between 2014 and 2016 using the Very Large Telescope Interferometer (Mérand et al. 2014) equipped with the PIONIER beam combiner (Berger et al. 2010; Le Bouquin et al. 2011). This instrument is operating in the infrared $H$ band $(\lambda=1.6 \mu \mathrm{m})$ using a spectral resolution of $R=40$. The four relocatable $1.8 \mathrm{~m}$ Auxiliary Telescopes (ATs) were positioned at stations A1-G1-J3-K0 or A0-G1-J2-J3 ${ }^{1}$. These quadruplets offer the longest available baselines (up to $140 \mathrm{~m}$ ), which are necessary to resolve the apparent disk of RS Pup $(\theta \approx 0.9$ mas) sufficiently well. The pointings of RS Pup were interspersed with observations of calibrator stars to estimate the interferometric transfer function of the instrument (Table 1). These calibrators were selected close angularly to RS Pup in order to minimize any possible bias caused by polarimetric mismatch of the beams. The raw data have been reduced using the pndrs data reduction software of PIONIER (Le Bouquin et al. 2011), which produces calibrated squared visibilities and phase closures. Two examples of the measured RS Pup squared visibilities are presented in Fig. 1. The visibilities were classically converted into uniform disk (UD) angular diameters (see, e.g., Mozurkewich et al. 2003; and Young 2003) that are listed in Table 2.

\subsection{Photometry}

As the measurements available in the literature are of uneven quality for RS Pup, we obtained new photometry in the JohnsonKron-Cousins BVR system using the ANDICAM CCD camera on the SMARTS ${ }^{2} 1.3 \mathrm{~m}$ telescope at Cerro Tololo Interamerican Observatory (CTIO). A total of 277 queue-scheduled observations were made by service observers between 2008 February 28 and 2011 January 25 . The exposure times were usually one second in each filter, but nevertheless, many of the $V$ images and most of the $R$ images were saturated, especially around maximum light, and had to be discarded. After standard flat-field corrections of the frames, we determined differential magnitudes relative to a nearby comparison star. In order to convert

\footnotetext{
1 https://www . eso.org/paranal/telescopes/vlti/ configuration/

2 SMARTS is the Small \& Moderate Aperture Research Telescope System; http: //www . astro.yale. edu/smarts
} 
P. Kervella et al.: Projection factor of the long-period Cepheid RS Pup
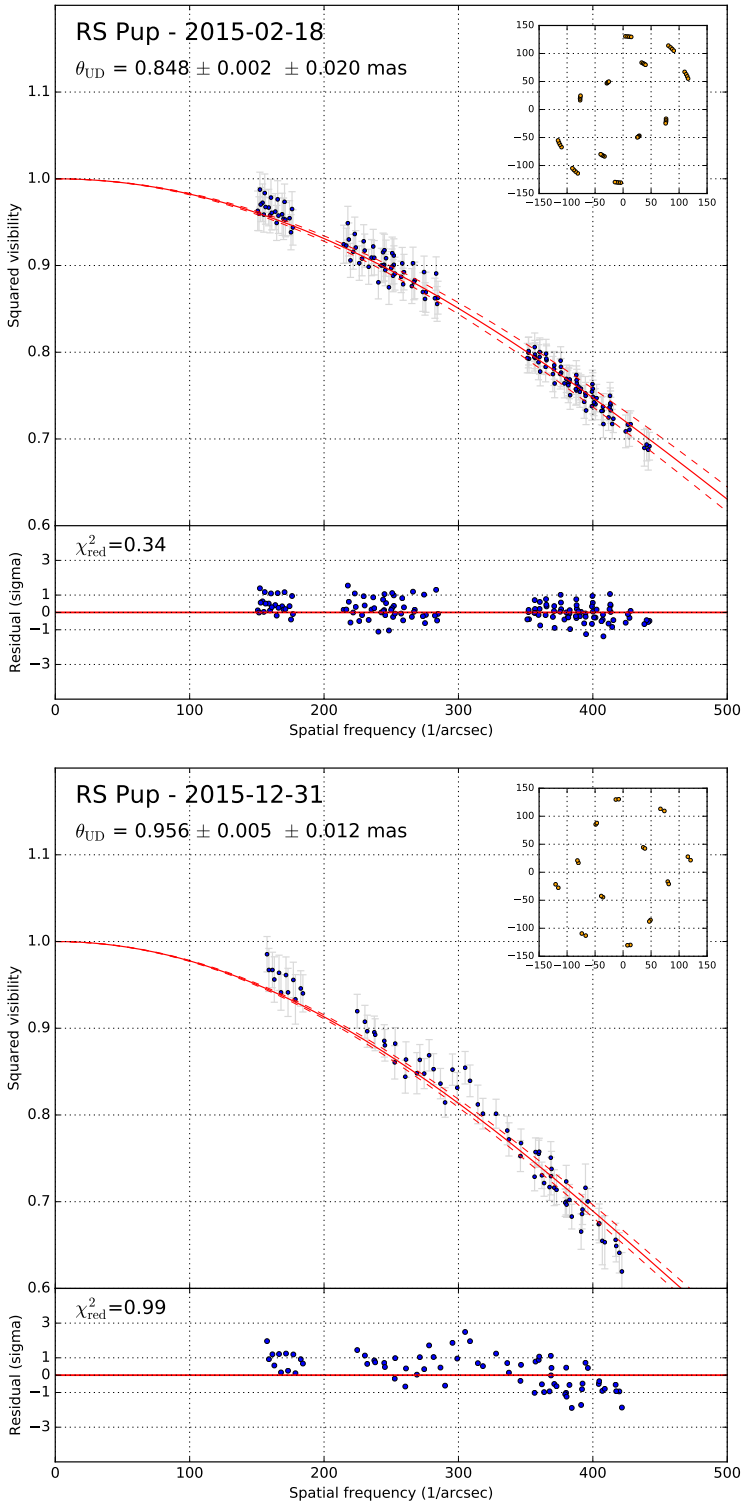

Fig. 1. Examples of PIONIER squared visibilities collected on RS Pup on the night of 18 February 2015, close to the minimum angular diameter phase (top panel), and on 31 December 2015, close to maximum angular diameter (bottom panel). The solid line is the best-fit uniform disk visibility model, and the dashed lines represent the limits of the $\pm 1 \sigma$ uncertainty domain on the angular diameter. The $(u, v)$ plane coverage is shown in the upper right subpanels, with axes labeled in meters.

the relative magnitudes into calibrated values, the $B V R$ magnitudes of the comparison star were determined through observations of Landolt (1992) standard-star fields obtained on seven photometric nights. The resulting $B V R$ light curves are presented in Fig. 2, phased with a period $P=41.5113$ days and $T_{0}\left[\mathrm{JD}_{\odot}\right]=2455501.254$. As discussed further in this section, this period is suitable over the range of the SMARTS observing epochs (2008-2011). The list of measured magnitudes is given in Table A.2. The associated uncertainty is estimated to \pm 0.03 mag per measurement (Winters et al. 2011).

We supplemented the new SMARTS photometric measurements with archival visible light photometry from Moffett \& Barnes (1984), Berdnikov (2008), and Pel (1976). In order to improve the coverage of the recent epochs, we also added a set of accurate photoelectric measurements retrieved
Table 2. PIONIER observations of RS Pup.

\begin{tabular}{cccc}
\hline \hline UT Date & MJD & Cal. & $\begin{array}{c}\theta_{\text {UD }} \pm \sigma_{\text {stat. }} \pm \sigma_{\text {syst. }} \\
\text { mas })\end{array}$ \\
\hline $2014-04-03$ & 56750.0178 & 1,2 & $0.860 \pm 0.011 \pm 0.020$ \\
$2014-05-08$ & 56785.0009 & 1,2 & $0.801 \pm 0.007 \pm 0.020$ \\
$2015-01-15$ & 57037.3056 & 1,2 & $0.813 \pm 0.005 \pm 0.020$ \\
$2015-01-16$ & 57038.3660 & 1,2 & $0.830 \pm 0.009 \pm 0.020$ \\
$2015-01-17$ & 57039.3643 & 1,2 & $0.882 \pm 0.015 \pm 0.020$ \\
$2015-02-14$ & 57067.1757 & 1,2 & $0.916 \pm 0.005 \pm 0.020$ \\
$2015-02-18$ & 57071.0997 & 1,2 & $0.848 \pm 0.002 \pm 0.020$ \\
$2015-02-21$ & 57074.1467 & 1,2 & $0.827 \pm 0.010 \pm 0.020$ \\
$2015-12-27$ & 57383.1893 & 3,4 & $0.983 \pm 0.007 \pm 0.012$ \\
$2015-12-31$ & 57387.1858 & 3,4 & $0.956 \pm 0.005 \pm 0.012$ \\
$2016-02-21$ & 57439.1475 & 3,4 & $0.933 \pm 0.011 \pm 0.012$ \\
\hline
\end{tabular}

Notes. We list the mean modified Julian date (MJD) of each observing night, the calibrator stars, the uniform disk diameter adjusted on the squared visibility measurements, and its statistical and systematic (calibration) uncertainties.

SMARTS 1.3m Photometry of RS Pup 2008-2011

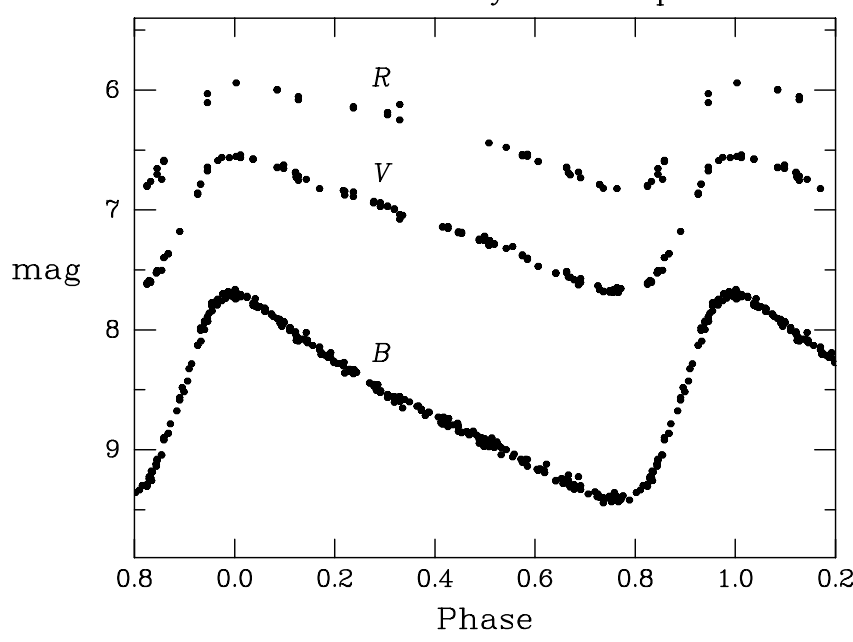

Fig. 2. SMARTS light curves of RS Pup in Johnson $B, V$, and Kron-Cousins $R$.

from the AAVSO database ${ }^{3}$. These recent measurements in the Johnson $V$ band are listed in Table A.3 and plotted in Fig. A.1. They cover the JD range 2456400 (April 2013) to 2457550 (June 2016), which matches our PIONIER interferometric observations well. Finally, we also included in our dataset the near-infrared $J H K$ band photometry from Laney \& Stobie (1992) and Welch et al. (1984).

\subsection{Radial velocities}

We included in our dataset the radial velocity measurements from Anderson (2014) that provide an excellent coverage of several pulsation cycles of RS Pup with a high accuracy. We complemented these data with the measurements obtained by Storm et al. (2004). As discussed by Anderson (2014), the radial velocity curve of RS Pup is not perfectly reproduced cycleto-cycle. This is potentially a difficulty for the application of

3 https://WWW.aavso.org 

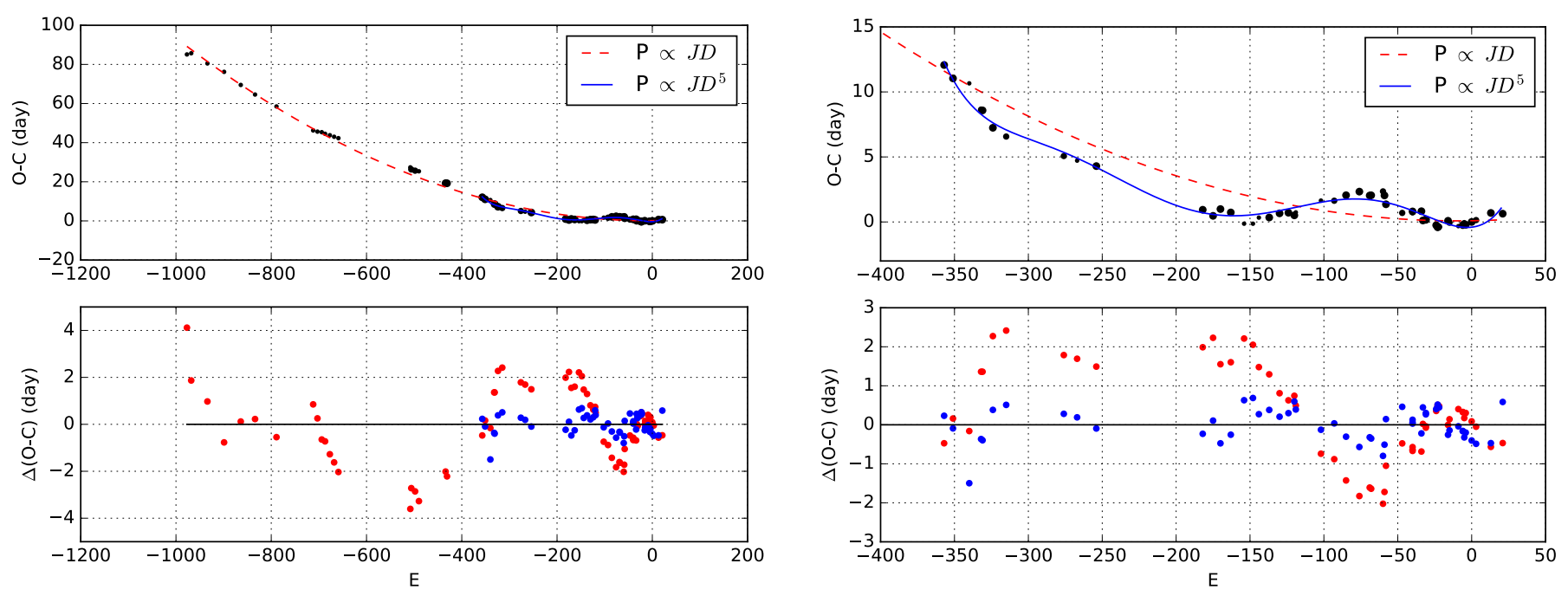

Fig. 3. Left column: O-C diagram for RS Pup (top panel) and residuals of the model (bottom panel) for a linear period variation (red dashed line and points) and a fifth-degree polynomial function (blue solid line and points). The size of the black points in the upper panel is proportional to their weight in the fit (Table A.1). Right column: enlargement of the O-C diagram covering the last 400 pulsation cycles of RS Pup.

the BW technique, which relies on observational datasets that are generally obtained at different epochs and therefore different pulsation cycles. This induces an uncertainty on the amplitude of the linear radius variation, and therefore on the derived parameters (distance or $p$-factor). Following the approach by Anderson et al. (2016b), we estimate in Sect. 3.1 the uncertainty induced on the $p$-factor by separately fitting the different cycles monitored by Anderson (2014).

\subsection{Phasing of the datasets}

We took particular care to properly phase the different datasets, a task that is complicated by the rapidly changing period of RS Pup. This is an important step in the fitting process, however, as an incorrect phasing results in biases on the derived model parameters.

As a first-order approach, the pulsation period $P$ and its linear rate of variation have been determined with the classical method of the O-C diagram (Sterken 2005). The diagram constructed for the moments of the maximum brightness covering more than a century is shown in the left panel of Fig. 3. The relevant data used for constructing the $\mathrm{O}-\mathrm{C}$ diagram are listed in Table A.1. The general trend of the period variation is an increase, with a superimposed oscillation exhibiting a pseudo-period on the order of three decades. When calculating the $\mathrm{O}-\mathrm{C}$ values, the reference epoch $E=0$ was taken as $\mathrm{JD}_{\odot} 2455$ 501.254. This is the normal maximum determined from the SMARTS light curve shown in Fig. 2. The variable $E$ designates the number of pulsation cycles that occurred since this reference epoch. The initial pulsation period was arbitrarily taken as 41.49 days. The second-order weighted least-squares fit to the $\mathrm{O}-\mathrm{C}$ residuals is also plotted in Fig. 3. The equation of the fitted parabola is (expressed in Julian date)

$$
\begin{aligned}
C= & 2455501.3428 \pm 0.1756 \\
& +(41.491734 \pm 0.001404) \times E \\
& +\left(9.51510^{-5} \pm 1.73310^{-5}\right) \times E^{2}
\end{aligned}
$$

As the $E^{2}$ coefficient in this equation is positive, the parabola in the $\mathrm{O}-\mathrm{C}$ diagram tends toward positive values, thus indicating that the period is increasing with time. Both the $\mathrm{O}-\mathrm{C}$ graph and the parabolic fit are in good agreement with their counterpart obtained by Berdnikov et al. (2009), who find a secular period change of $7.82410^{-5} \pm 1.968 \times 10^{-5}$ (quadratic term, expressed in fraction of the period per cycle). The secular period increase that we derive corresponds to a lengthening of +0.1675 day over a century, or $+144.7 \mathrm{~s} / \mathrm{yr}$. This value is high, but not without precedent among long-period classical Cepheids (Mahmoud \& Szabados 1980). This rate of secular period change corresponds to the expected value for a third crossing Cepheid with a period like RS Pup (Anderson et al. 2016c).

The erratic period changes superimposed on the monotonic period variation of RS Pup are clearly seen on the residuals of the $\mathrm{O}-\mathrm{C}$ fit in Fig. 3. In the bottom panels of this figure, the parabola has been subtracted from the $\mathrm{O}-\mathrm{C}$ values listed in Table A.1 (as shown in the upper panels). There are three intervals in this diagram where the pulsation period can be approximated with a constant value: between 1995 and 2002 as $41.518 \pm$ 0.002 days, between 2003 and 2007 as $41.437 \pm 0.002$ days, and between 2008 and 2013 as $41.512 \pm 0.002$ days. Kervella et al. (2014) adopted a period $P=41.5117$ days for the epoch of the HST/ACS observations (2010) that were used to estimate the distance of RS Pup through its light echoes. It is worth noting that the scatter between the subsequent data points can be intrinsic to the stellar pulsation: this phenomenon is interpreted as a cycle-to-cycle jitter in the pulsation period, as observed in V1154 Cyg, the only Cepheid in the original Kepler field (Derekas et al. 2012). It was proposed by Neilson \& Ignace (2014) that the physical mechanism underlying the period jitter of V1154 Cyg is linked to the presence of convective hot spots on the photosphere of the star. This explanation may also apply to RS Pup, whose relatively low effective temperature could favor the appearance of such convective features.

The period changes that occurred in the past few decades induced a variability of the maximum light epochs of 3 to 4 days, that is, up to 0.10 in phase shift. Such a large phase shift would degrade the quality of the SPIPS combined fit of the observables, in particular the photometry that is spread over four decades. To take the period changes into account, we adopt a polynomial model of degree five. This relatively high degree allows us to fit the observed epochs of maximum light much better than the linear model, as shown in the residuals of the O-C diagram (Fig. 3, 


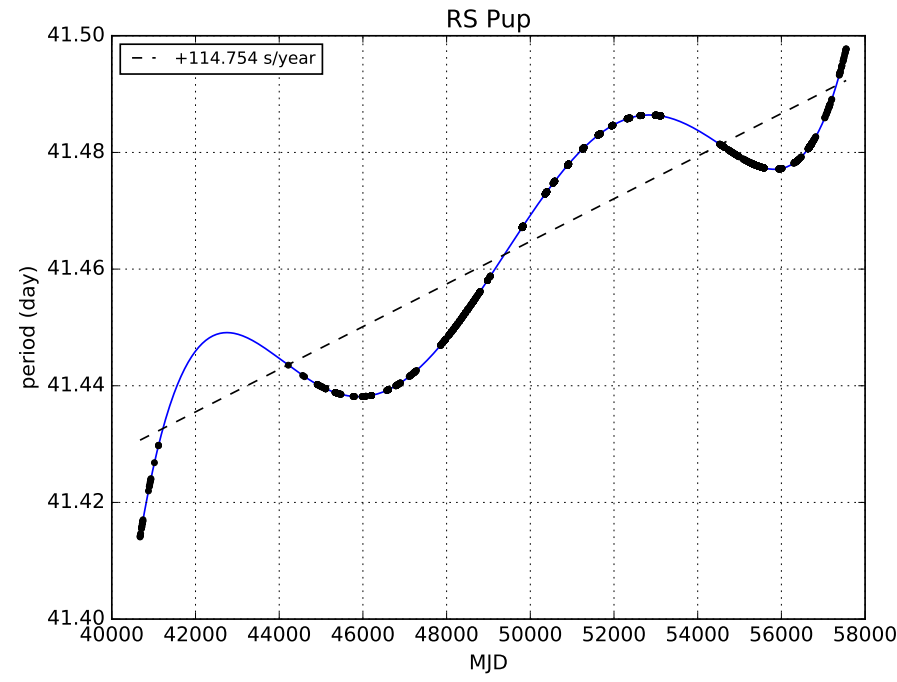

Fig. 4. Polynomial fit of the changing period of RS Pup. The blue curve is a degree-five polynomial fit of the period values (black points). The black dashed line represents the linear trend of the period change over the past $50 \mathrm{yr}$.

bottom panels). The period in days as a function of the observing epoch $T$ (expressed in modified Julian date) is given by the polynomial expression

$$
\begin{aligned}
P(\mathrm{MJD})= & (41.438138 \pm 0.00070)-3.42244 \times 10^{-7}(\Delta t) \\
& +0.23085 \times 10^{-8}(\Delta t)^{2}+0.13219 \times 10^{-12}(\Delta t)^{3} \\
& -0.74919 \times 10^{-16}(\Delta t)^{4}+0.42849 \times 10^{-20}(\Delta t)^{5},
\end{aligned}
$$

with $\Delta t=\mathrm{MJD}-\mathrm{MJD}_{0}$ the number of days since the reference epoch $\mathrm{MJD}_{0}=45838.0313$. The $+114.8 \mathrm{~s} /$ year linear rate of the period change over the past $50 \mathrm{yr}$ shown in Fig. 4 is close to the value obtained from the fit of the complete dataset with a linearly variable period $(+144.7 \mathrm{~s} /$ year $)$.

\section{Analysis of RS Pup using SPIPS}

The SPIPS modeling code (Mérand et al. 2015) considers a pulsating star as a sphere with a changing effective temperature and radius, over which is superimposed a combination of atmospheric models from precomputed grids (ATLAS9). The presence of a circumstellar envelope emitting in the infrared $K$ and $H$ bands is included in the model, as is the interstellar reddening. The best-fit SPIPS model of RS Pup is presented in Fig. 5 together with the observational data, and the corresponding bestfit parameters are listed in Table 3. The quality of the fit is generally very good for all observing techniques, and the phasing of the different datasets is satisfactory. The interpolation of the radial velocity curve was achieved using splines with optimized node positions. We assume the distance $d=1910 \pm 80 \mathrm{pc}$ determined by Kervella et al. (2014) as a fixed parameter in this fit.

\subsection{Projection factor}

Considering the complete radial velocity data set, we obtain a projection factor of $p=1.250$ with a statistical uncertainty from the fit of $\sigma_{\text {stat }}= \pm 0.034$.
The primary source of systematic error on $p$ is the uncertainty on the adopted light echo distance. As the $p$-factor and the distance are fully degenerate parameters, the $\pm 4.2 \%$ distance error bar directly translates into a $\sigma_{\text {dist }}= \pm 0.053$ uncertainty on $p$.

As shown by Anderson (2014, 2016), the cycle-to-cycle repeatability of the velocity curve of long-period Cepheids is imperfect. Anderson et al. (2016b) demonstrated that for $\ell$ Car, variations of the $p$-factor of $5 \%$ are observed between cycles. To quantify this effect for RS Pup, we adjusted distinct SPIPS models on the four cycles sampled by Anderson (2014). The results are shown in Figs. B.1 to B.4. We observe a standard deviation of $\sigma=0.028$ over the four $p$-factor values derived for the different cycles that we translate into a systematic uncertainty of $\sigma_{\text {cycle }}= \pm 0.014$ on the $p$-factor. The SPIPS models resulting from the separate fit of the radial velocity datasets of Storm et al. (2004) and Anderson (2014) are presented in Figs. B.5 and B.6, respectively. The derived $p$-factors from these two datasets do not show any significant bias beyond $\sigma_{\text {cycle }}$.

We assumed in the SPIPS model that the $p$-factor is constant during the pulsation cycle of the star. This is a simplification, as the $p$-factor is proportional to the limb darkening, which is known to change with the effective temperature of the star. The amplitude of the $p$-factor variation induced by the changing limb darkening is expected to be small. The effective temperature of RS Pup changes by $1300 \mathrm{~K}$ during its pulsation (4600-5900 K, Fig. 5 and Table 3). Neilson \& Lester (2013) presented predictions of the limb-darkening corrections applicable to interferometric angular diameter measurements based on a spherical implementation of Kurucz's ATLAS models. For the temperature range of RS Pup considering $\log g \approx 1.0$ and $\left.M \approx 10 M_{\odot}\right)$, the listed correction factor $k=\theta_{\mathrm{UD}} / \theta_{\mathrm{LD}}$ in the $V$ band (in which the spectroscopic measurements are obtained) ranges from $k_{V}=0.9116(4600 \mathrm{~K})$ to $k_{V}=0.9161(5900 \mathrm{~K})$ over the cycle. We consider here that this variation of $0.5 \%$ is negligible compared to the other sources of systematic uncertainty (distance and cycle-to-cycle variations).

In summary, combining the systematic uncertainties through $\sigma_{\text {syst }}=\left(\sigma_{\text {dist }}^{2}+\sigma_{\text {cycle }}^{2}\right)^{1 / 2}$, we obtain the $p$-factor of RS Pup for the cross-correlation radial velocity method:

$p=1.250 \pm 0.034 \pm 0.054=1.250 \pm 0.064( \pm 5.1 \%)$.

\subsection{Color excess and circumstellar envelope}

We derive a color excess $E(B-V)=0.4961 \pm 0.0060$, higher than the value obtained by Fouqué et al. (2007), who list $E(B-V)=$ $0.457 \pm 0.009$ for RS Pup. The possible presence of an excess emission in the infrared $K(\lambda \approx 2.2 \mu \mathrm{m})$ and $H(\lambda \approx 1.6 \mu \mathrm{m})$ bands is adjusted as a parameter by the SPIPS code. For RS Pup, we detect a moderately significant excess emission of $\Delta m_{K}=$ $0.027 \pm 0.011$ in the $K$ band, and marginal in the $H$ band $\left(\Delta m_{H}=0.016 \pm 0.011 \mathrm{mag}\right)$. This low level of excess emission is in agreement with Kervella et al. (2009), who did not detect a photometric excess in the $K$ band, although a considerable excess flux is found in the thermal infrared $(10 \mu \mathrm{m})$ and at longer wavelengths. We note that the best-fit infrared excess values for the different pulsation cycles of RS Pup (Figs. B.1 to B.6) are consistent within a few millimagnitudes.

\subsection{Limit on the presence of companions}

We checked for the presence of a companion in the PIONIER interferometric data using the companion analysis and non-detection in interferometric data algorithm (CANDID, 

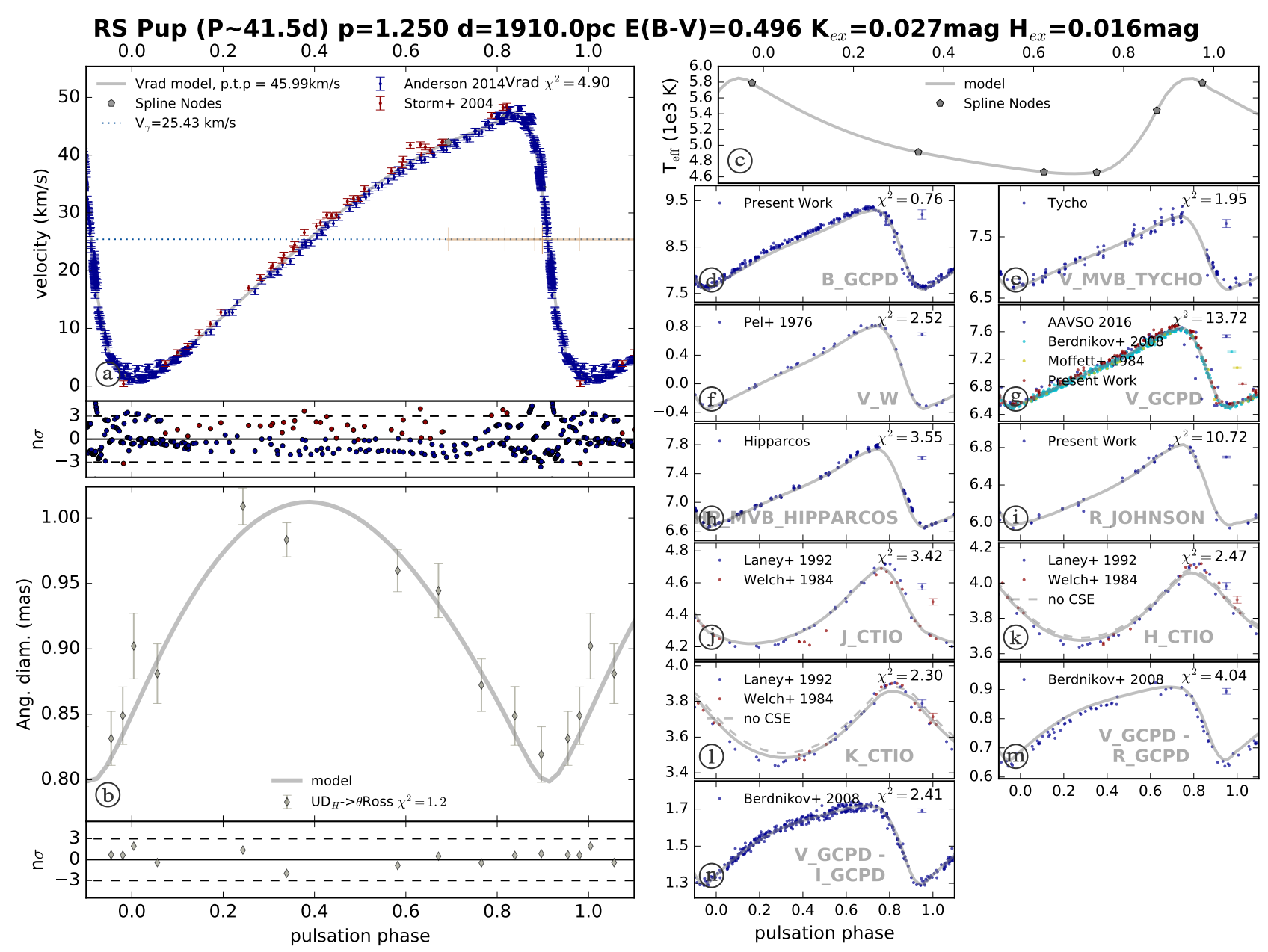

Fig. 5. SPIPS combined fit of the observations of RS Pup.

Gallenne et al. 2015). The interferometric observables are particularly sensitive to the presence of companions down to high contrast ratios and small separations, as demonstrated, for instance, by Absil et al. (2011), Gallenne et al. (2013, 2014). We did not detect any secondary source, ruling out the presence of a stellar companion with a contrast in the $H$ band less than approximately 6 magnitudes (flux ratio $f / f_{\text {Cepheid }}=0.4 \%$ ) within 40 mas of the Cepheid (Fig. 6).

The $\gamma$-velocity of RSPup measured using the crosscorrelation technique is presented in Fig. 7, and the values are listed in Table 4. The cycle-to-cycle random variation of the amplitude of the radial velocity of RS Pup (Anderson 2014) may induce systematic uncertainties on the determination of the $\gamma$-velocity. This will particularly be the case if the radial velocity phase coverage is incomplete. For this reason, while the amplitude of the fluctuations appears significant, it is difficult to conclude that it is caused by a companion. It is interesting to note that the $\gamma$-velocity value depends on the technique used for the radial velocity measurement: Nardetto et al. (2008) find a $\gamma$-velocity of $v_{\gamma}=-25.7 \pm 0.2 \mathrm{~km} \mathrm{~s}^{-1}$ for RS Pup after correction of the $\gamma$-asymmetry of its spectral lines. The $\gamma$-velocity can also depend on which lines are included in the cross-correlation mask.

\section{Discussion}

For a review of the current open questions related to the $p$-factor, in particular in the context of the interferometric version of the BW technique, we refer to Barnes $(2009,2012)$.
A summary of the available predictions and measurements of the $p$-factors of RSPup and of the similar long-period Cepheid $\ell$ Car is presented in Table 5. Most authors based their BW distance determination on the linear period- $p$-factor relation established by Hindsley \& Bell (1986, 1989): $p=$ $1.39-0.03 \log P$. Owing to the weak dependence on period, the $p$-factors predicted for RS Pup and $\ell$ Car by this relation are both very close to $p=1.34$. The theoretical calibration of the period$p$-factor $(P p)$ relation by Neilson et al. (2012) gives a geometric $p$-factor of $p_{0}=[1.402 \pm 0.002]-[0.0440 \pm 0.0015] \log P$ ( $V$ band, spherical model, linear law), to be multiplied by the period-dependent velocity gradient and differential velocity corrections introduced by Nardetto et al. (2007). The recent work by Nardetto et al. (2014a) including $\delta$ Scuti stars confirms the $P p$ relation by Nardetto et al. (2009) and proposes a common $P p$ relation between Cepheids and $\delta$ Scuti stars $(p=[1.31 \pm 0.01]-$ $[0.08 \pm 0.01] \log P)$. The Nardetto et al. (2014a) relation yields $p=1.181$ for RS Pup and $p=1.186$ for $\ell$ Car. The relation from Storm et al. (2011) is much steeper $(p=[1.550 \pm 0.04]-$ $[0.186 \pm 0.06] \log P)$. Groenewegen (2007) used the Cepheid trigonometric parallaxes from Benedict et al. (2007) to derive a $P p$ relation of the form $p=[1.28 \pm 0.15]-[0.01 \pm 0.16] \log P$, which is consistent with a constant $p$-factor with $p=1.27 \pm 0.05$.

Figure 8 gives an overview of the available measurements of $p$-factors of Cepheids, including the Type II Cepheid $\kappa$ Pav (Breitfelder et al. 2015). We selected for this plot the $p$-factor values with a relative accuracy better than $10 \%$. We removed from the sample the binary Cepheid FFAql for which the 
Table 3. Parameters of the SPIPS model of RS Pup.

\begin{tabular}{ll}
\hline \hline Parameter & Value $\pm \sigma_{\text {stat }} \pm \sigma_{\text {syst }}$ \\
\hline$\theta_{0}(\mathrm{mas})^{a}$ & $0.8490 \pm 0.0034 \pm 0.0120$ \\
$\langle\theta\rangle(\mathrm{mas})^{a}$ & $0.9305 \pm 0.0034 \pm 0.0120$ \\
$v_{\gamma}\left(\mathrm{km} \mathrm{s}^{-1}\right)$ & $25.423 \pm 0.200$ \\
$E(B-V)$ & $0.4961 \pm 0.0060$ \\
$K$ excess & $0.027 \pm 0.011$ \\
$H$ excess & $0.016 \pm 0.011$ \\
$p$-factor & $1.250 \pm 0.034 \pm 0.054$ \\
MJD & $45838.0313 \pm 0.098$ \\
Period (days $)^{b}$ & $41.438138 \pm 0.00070$ \\
Period change $(\mathrm{s} / \text { year })^{c}$ & +114.8 \\
Distance $(\mathrm{pc})$ & $1910 \pm 80($ fixed $)$ \\
\hline Radius $\left(R_{\odot}\right)$ & $191(164 / 208)$ \\
Eff. temperature $(K)$ & $5060(4640 / 5850)$ \\
Bolom. luminosity $\left(L_{\odot}\right)$ & $21700(14200 / 29500)$ \\
Bolometric magnitude & $-6.072(-6.434 /-5.640)$ \\
\hline
\end{tabular}

Notes. The upper part of the table lists the primary model parameters, and the lower part gives derived physical parameters (mean value and minimum/maximum over the pulsation cycle). ${ }^{(a)} \theta_{0}$ is the limbdarkened disk (Rosseland) angular diameter at phase zero, and $\langle\theta\rangle$ the phase-average mean angular diameter over the pulsation cycle. ${ }^{(b)} \mathrm{Pe}$ riod at the reference epoch $\mathrm{MJD}_{0} .{ }^{(c)}$ Rate of period change as shown in Fig. 4.

HST/FGS distance is questionable (see the discussion, e.g., in Breitfelder et al. 2016 and Turner et al. 2013). As shown by Anderson et al. (2016a), the presence of a companion can bias the parallax. The weighted average of the selected measurements is $\bar{p}=1.293 \pm 0.039$, and the reduced $\chi^{2}$ of the measurements with respect to this constant value is $\chi_{\text {red }}^{2}=0.9$. If we include FF Aql in the sample, we obtain $\bar{p}=1.285$. The uncertainty of $\bar{p}$ was computed from the combination of the error bars of the independent measurements of OGLE-LMC-CEP$0227(P=3.80 \mathrm{~d}, p=1.21 \pm 0.05$, Pilecki et al. 2013), $\delta$ Cep $(P=5.37 \mathrm{~d}, p=1.288 \pm 0.054$, Mérand et al. 2015), and the present measurement of RS Pup $(P=41.5 \mathrm{~d}, p=1.250 \pm 0.064)$. We did not average the error bars of the different $p$-factor measurements from the HST/FGS distances as the degree of correlation between them and the possible associated systematics are uncertain. For the same reason, we did not average the uncertainties of the two $p$-factor measurements of binary Cepheids in the LMC from Pilecki et al. (2013) and Gieren et al. (2015), and we selected only the best $p$-factor of $\delta$ Cep derived by Mérand et al. (2015). In agreement with the present results, Breitfelder et al. (2016) also concluded from a fit to the complete sample of measured $p$-factors that a constant value of $\bar{p}=1.324 \pm 0.024(1 \sigma$ from our value) reproduces the measurements.

The good agreement of the constant $p$-factor model $\bar{p}=$ $1.293 \pm 0.039$ with the measurements indicates that this coefficient is mildly variable over a broad range of Cepheid periods (3.0 to 41.5 days). This result can be explained by the relatively narrow range of effective temperature and gravity of Cepheids, which results in a minor variation of their limb darkening. Neilson \& Lester (2013) predict changes of the limbdarkening coefficient $k=\theta_{\mathrm{UD}} / \theta_{\mathrm{LD}}$ of only a few percent in the $V$ band over the full range of classical Cepheid properties. The difference is even smaller at longer wavelengths. The spherical
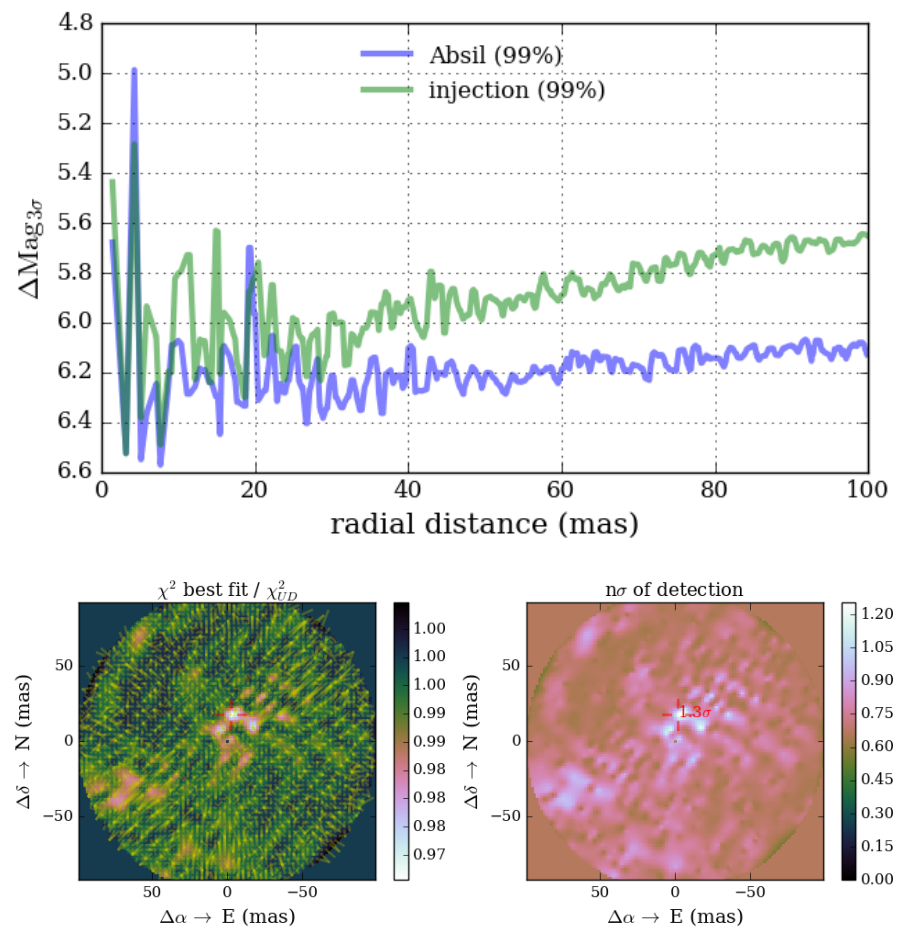

Fig. 6. Top panel: upper limit $(3 \sigma)$ of the flux contribution of companions of RS Pup as a function of the angular separation from the Cepheid. The limits obtained using the approaches of Absil et al. (2011) and Gallenne et al. (2015) are shown separately. Bottom panel: map of the $\chi^{2}$ of the best binary model fit (left) and statistical significance of the detection (right). No significant source is found in the field of view.

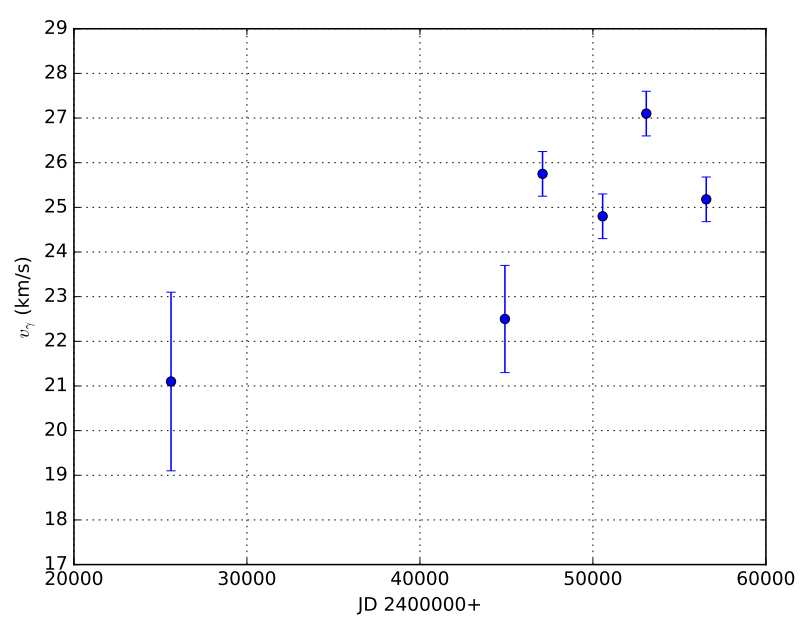

Fig. 7. Observed $\gamma$-velocity of RS Pup.

models in the $V$ band by these authors give $k=0.9337$ for the hottest phase of a short-period Cepheid $(7000 \mathrm{~K}, \log g=2.0$, $m=5 M_{\odot}$ ), less than $2.5 \%$ away from the value $k=0.9116$ obtained for the coolest phase of RS Pup $(4600 \mathrm{~K}, \log g=1.0$, $\left.m=10 M_{\odot}\right)$. The mild dependence of the $p$-factor on the period is consistent with the $P p$ relation proposed by Groenewegen (2007).

The precision of the parallaxes of the first data release of Gaia-TGAS (Lindegren et al. 2016) is too low to accurately determine the $p$-factor of nearby Cepheids (see, e.g., Casertano et al. 2017). The availability in 2018 of the second Gaia data release (Gaia Collaboration et al. 2016b) will provide 
Table 4. $\gamma$-velocities of RS Pup from the cross-correlation technique.

\begin{tabular}{lcl}
\hline \hline Mean JD & $v_{\gamma}\left(\mathrm{km} \mathrm{s}^{-1}\right)$ & Reference \\
\hline 2425612 & $21.1 \pm 2.0$ & Joy (1939) \\
2444913 & $22.5 \pm 1.2$ & Barnes et al. (1988) \\
2447090 & $25.8 \pm 0.5$ & Storm et al. (2004) \\
2450563 & $24.8 \pm 0.5$ & Bersier (2002) \\
2453085 & $27.1 \pm 0.5$ & Nardetto et al. (2009) \\
2456551 & $25.2 \pm 0.5$ & Anderson (2014) \\
\hline
\end{tabular}

Table 5. Measured (top section) $p$-factor values of RS Pup and $\ell$ Car and predictions from period- $p$-factor relations (bottom section).

\begin{tabular}{lcc}
\hline \hline Reference & RS Pup & $\ell$ Car \\
\hline Breitfelder et al. (2016) & - & $1.23 \pm 0.12$ \\
Anderson et al. (2016b) & $c$ & $1.27 \pm 0.12$ \\
Present work & - & - \\
\hline Burki et al. (1982) & $1.250 \pm 0.064$ & 1.36 \\
Hindsley \& Bell (1986) & 1.341 & 1.343 \\
Gieren et al. (2005) $_{\text {Groenewegen (2007) }}{ }^{c}$ & $1.337 \pm 0.038$ & $1.347 \pm 0.037$ \\
Laney \& Joner (2009) & $1.196 \pm 0.038$ & $1.201 \pm 0.036$ \\
Storm et al. (2011) & $1.249 \pm 0.105$ & $1.262 \pm 0.101$ \\
Neilson et al. (2012) & $1.140 \pm 0.003$ & $1.146 \pm 0.003$ \\
Groenewegen (2013) & $1.112 \pm 0.030$ & $1.128 \pm 0.030$ \\
Nardetto et al. (2014a) & $1.181 \pm 0.019$ & $1.186 \pm 0.018$ \\
\hline
\end{tabular}

Notes. ${ }^{(a)}$ Constant $p$-factor value; ${ }^{(b)}$ excluding FF Aql; ${ }^{(c)}$ average of the measured values.

very accurate parallaxes for hundreds of Galactic Cepheids, however, including RS Pup, which will be among the longest periods in the sample. The ongoing observations of a sample of 18 long-period Cepheids by Casertano et al. (2016) using the spatial scanning technique with the HST/WFC3 has started to provide accurate parallaxes with accuracies of $\pm 30 \mu$ s for these rare pulsators. At a later stage, accurate broadband epoch photometry will also be included in the Gaia data releases (see, e.g., Clementini et al. 2016). Combining Gaia data with archival observations, the SPIPS technique will enable a very accurate calibration of the $P p$ relation of Cepheids, and therefore of their distance scale, which is still today an essential ingredient in determining the local value of $H_{0}$ (Riess et al. 2016). For the nearest Cepheids of the Gaia and HST/WFC3 samples, the availability of interferometric angular diameters will significantly improve the quality of the determination of their parameters thanks to the resolution of the usual degeneracy between effective temperature and interstellar reddening. Even for distant Cepheids, however, whose angular diameters cannot be measured directly, the robustness of the SPIPS algorithm will enable an accurate calibration of their physical properties, including the $p$-factor, once their parallaxes are known.

Acknowledgements. We would like to thank Vello Tabur and Stanley Walker for sending us their unpublished photometric observations, and Marcella Marconi. We acknowledge with thanks the variable star observations from the AAVSO International Database contributed by observers worldwide and used in this research. The authors acknowledge the support of the French Agence Nationale de la Recherche (ANR), under grant ANR-15-CE31-0012-01 (project UnlockCepheids). P.K., A.G., and W.G. acknowledge support of the French-Chilean exchange program ECOS-Sud/CONICYT (C13U01). W.G. and G.P. gratefully

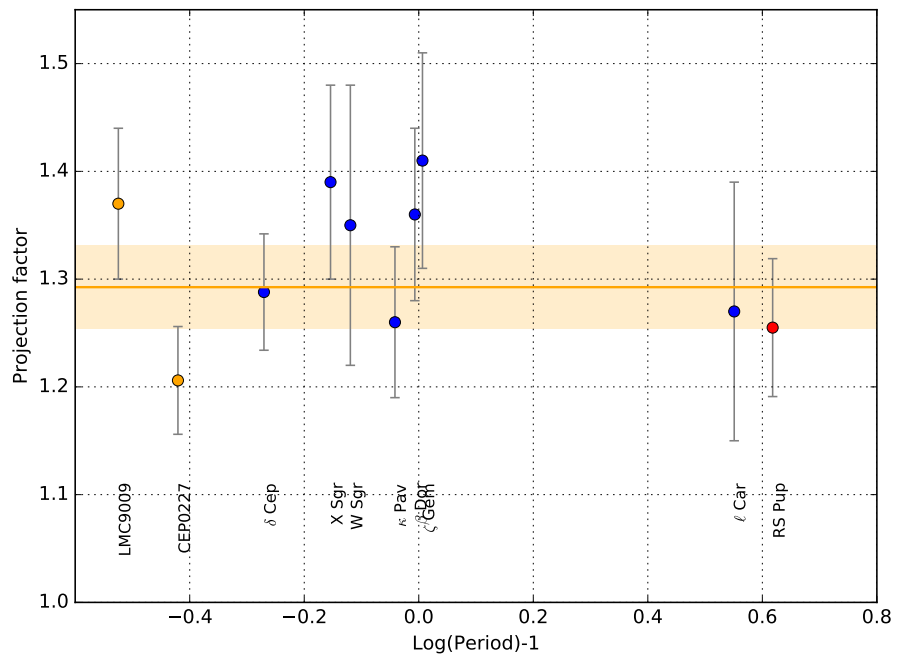

Fig. 8. Distribution of the measured $p$-factors of Cepheids with better than $10 \%$ relative accuracy. The references for the different measurements (except RS Pup) are listed in Breitfelder et al. (2016). The blue points use HST-FGS distances (Benedict et al. 2002, 2007), the orange points are the LMC eclipsing Cepheids (Pilecki et al. 2013; Gieren et al. 2015). The solid line and orange shaded area represent the weighted average $\bar{p}=1.293 \pm 0.039$.

acknowledge financial support for this work from the BASAL Centro de Astrofisica y Tecnologias Afines (CATA) PFB-06/2007. W.G. also acknowledges financial support from the Millenium Institute of Astrophysics (MAS) of the Iniciativa Cientifica Milenio del Ministerio de Economia, Fomento y Turismo de Chile, project IC120009. We acknowledge financial support from the Programme National de Physique Stellaire (PNPS) of CNRS/INSU, France. L.S.z. acknowledges support from the ESTEC Contract No. 4000106398/12/NL/KML. The research leading to these results has received funding from the European Research Council (ERC) under the European Union's Horizon 2020 research and innovation programme (grant agreement No.695099). This research made use of Astropy ${ }^{4}$, a community-developed core Python package for Astronomy (Astropy Collaboration et al. 2013). This work has made use of data from the European Space Agency (ESA) mission Gaia (http://www. cosmos.esa.int/ gaia), processed by the Gaia Data Processing and Analysis Consortium (DPAC, http://www. cosmos.esa.int/web/gaia/dpac/consortium). Funding for the DPAC has been provided by national institutions, in particular the institutions participating in the Gaia Multilateral Agreement. We used the SIMBAD and VIZIER databases at the CDS, Strasbourg (France), and NASA's Astrophysics Data System Bibliographic Services.

\section{References}

Absil, O., Le Bouquin, J.-B., Berger, J.-P., et al. 2011, A\&A, 535, A68 Anderson, R. I. 2014, A\&A, 566, L10

Anderson, R. I. 2016, MNRAS, 463, 1707

Anderson, R. I., Casertano, S., Riess, A. G., et al. 2016a, ApJS, 226, 18 Anderson, R. I., Mérand, A., Kervella, P., et al. 2016b, MNRAS, 455, 4231 Anderson, R. I., Saio, H., Ekström, S., Georgy, C., \& Meynet, G. 2016c, A\&A, 591, A8

Astropy Collaboration, Robitaille, T. P., Tollerud, E. J., et al. 2013, A\&A, 558, A33

Barnes, T. G. 2009, in AIP Conf. Ser, 1170, eds. J. A. Guzik, \& P. A. Bradley, 3 Barnes, III, T. G. 2012, JAAVSO, 40, 256

Barnes, III, T. G., Moffett, T. J., \& Slovak, M. H. 1988, ApJS, 66, 43

Benedict, G. F., McArthur, B. E., Fredrick, L. W., et al. 2002, AJ, 124, 1695

Benedict, G. F., McArthur, B. E., Feast, M. W., et al. 2007, AJ, 133, 1810

Berdnikov, L. N. 1995, in IAU Colloq. 155, Astrophysical Applications of Stellar

Pulsation, eds. R. S. Stobie, \& P. A. Whitelock, ASP Conf. Ser., 83, 349

Berdnikov, L. N. 2008, VizieR Online Data Catalog: II/285

Berdnikov, L. N., Henden, A. A., Turner, D. G., \& Pastukhova, E. N. 2009, Astron. Lett., 35, 406

Berger, J.-P., Zins, G., Lazareff, B., et al. 2010, in SPIE Conf. Ser., 7734

Bersier, D. 2002, ApJS, 140, 465

4 Available at http://www . astropy.org/ 
Breitfelder, J., Kervella, P., Mérand, A., et al. 2015, A\&A, 576, A64 Breitfelder, J., Mérand, A., Kervella, P., et al. 2016, A\&A, 587, A117 Burki, G., Mayor, M., \& Benz, W. 1982, A\&A, 109, 258

Casertano, S., Riess, A. G., Anderson, J., et al. 2016, ApJ, 825, 11

Casertano, S., Riess, A. G., Bucciarelli, B., \& Lattanzi, M. G. 2017, A\&A, 599, A67

Clementini, G., Ripepi, V., Leccia, S., et al. 2016, A\&A, 595, A133

Dean, J. F., Cousins, A. W. J., Bywater, R. A., \& Warren, P. R. 1977, Mem. R. Astron. Soc., 83, 69

Derekas, A., Szabó, G. M., Berdnikov, L., et al. 2012, MNRAS, 425, 1312

Eggen, O. J., Gascoigne, S. C. B., \& Burr, E. J. 1957, MNRAS, 117, 406

ESA, ed. 1997, ESA SP 1200, The HIPPARCOS and TYCHO catalogues. Astrometric and photometric star catalogues derived from the ESA HIPPARCOS Space Astrometry Mission

Fouqué, P., Arriagada, P., Storm, J., et al. 2007, A\&A, 476, 73

Gaia Collaboration (Brown, A. G. A., et al.) 2016a, A\&A, 595, A2

Gaia Collaboration (Prusti, T., et al.) 2016b, A\&A, 595, A1

Gallenne, A., Monnier, J. D., Mérand, A., et al. 2013, A\&A, 552, A21

Gallenne, A., Mérand, A., Kervella, P., et al. 2014, A\&A, 561, L3

Gallenne, A., Mérand, A., Kervella, P., et al. 2015, A\&A, 579, A68

Gerasimovic, B. P. 1927, Harvard College Observatory Bulletin, 848, 14

Gieren, W., Pilecki, B., Pietrzyński, G., et al. 2015, ApJ, 815, 28

Gieren, W., Storm, J., Barnes, III, T. G., et al. 2005, ApJ, 627, 224

Groenewegen, M. A. T. 2007, A\&A, 474, 975

Groenewegen, M. A. T. 2013, A\&A, 550, A70

Harris, H. C. 1980, Ph.D. Thesis, Washington Univ., Seattle, USA

Hindsley, R., \& Bell, R. A. 1986, PASP, 98, 881

Hindsley, R. B., \& Bell, R. A. 1989, ApJ, 341, 1004

Innes, R. T. A., \& Gill, D. 1903, Annals of the Cape Observatory, 9, 1

Irwin, J. B. 1961, ApJS, 6, 253

Joy, A. H. 1939, ApJ, 89, 356

Kervella, P., Thévenin, F., Di Folco, E., \& Ségransan, D. 2004, A\&A, 426, 297

Kervella, P., Mérand, A., \& Gallenne, A. 2009, A\&A, 498, 425

Kervella, P., Bond, H. E., Cracraft, M., et al. 2014, A\&A, 572, A7

Lafrasse, S., Mella, G., Bonneau, D., et al. 2010a, VizieR Online Data Catalog: II/300

Lafrasse, S., Mella, G., Bonneau, D., et al. 2010b, in Proc. SPIE, 7734, Optical and Infrared Interferometry II, 77344E-77344E-11

Landolt, A. U. 1992, AJ, 104, 340

Laney, C. D., \& Stobie, R. S. 1992, A\&AS, 93, 93

Laney, C. D., \& Joner, M. D. 2009, in AI eds. J. A. Guzik, \& P. A. Bradley, Conf. Ser., 1170, 93

Le Bouquin, J.-B., Berger, J.-P., Lazareff, B., et al. 2011, A\&A, 535, A67

Leavitt, H. S. 1908, Annals of Harvard College Observatory, 60, 87
Leavitt, H. S., \& Pickering, E. C. 1912, Harvard College Observatory Circular, 173,1

Lindegren, L., Lammers, U., Bastian, U., et al. 2016, A\&A, 595, A4

Madore, B. F. 1975, ApJS, 29, 219

Mahmoud, F., \& Szabados, L. 1980, IBVS, 1895, 1

Mérand, A., Bordé, P., \& Coudé du Foresto, V. 2005a, A\&A, 433, 1155

Mérand, A., Kervella, P., Coudé du Foresto, V., et al. 2005b, A\&A, 438, L9

Mérand, A., Abuter, R., Aller-Carpentier, E., et al. 2014, in Optical and Infrared Interferometry IV, Proc. SPIE, 9146, 91460J

Mérand, A., Kervella, P., Breitfelder, J., et al. 2015, A\&A, 584, A80

Mitchell, R. I., Iriarte, B., Steinmetz, D., \& Johnson, H. L. 1964, Boletin de los Observatorios Tonantzintla y Tacubaya, 3, 153

Moffett, T. J., \& Barnes, III, T. G. 1984, ApJS, 55, 389

Mozurkewich, D., Armstrong, J. T., Hindsley, R. B., et al. 2003, AJ, 126, 2502

Nardetto, N., Mourard, D., Mathias, P., Fokin, A., \& Gillet, D. 2007, A\&A, 471, 661

Nardetto, N., Stoekl, A., Bersier, D., \& Barnes, T. G. 2008, A\&A, 489, 1255

Nardetto, N., Gieren, W., Kervella, P., et al. 2009, A\&A, 502, 951

Nardetto, N., Poretti, E., Rainer, M., et al. 2014a, A\&A, 561, A151

Nardetto, N., Storm, J., Gieren, W., Pietrzyński, G., \& Poretti, E. 2014b, IAU Symp. 301, eds. J. A. Guzik, W. J. Chaplin, G. Handler, \& A. Pigulski, 145

Neilson, H. R., \& Ignace, R. 2014, A\&A, 563, L4

Neilson, H. R., \& Lester, J. B. 2013, A\&A, 554, A98

Neilson, H. R., Nardetto, N., Ngeow, C.-C., Fouqué, P., \& Storm, J. 2012, A\&A, 541, A 134

Pel, J. W. 1976, A\&AS, 24, 413

Pilecki, B., Graczyk, D., Pietrzyński, G., et al. 2013, MNRAS, 436, 953

Pojmanski, G. 2002, Acta Astron., 52, 397

Riess, A. G., Macri, L., Casertano, S., et al. 2011, ApJ, 730, 119

Riess, A. G., Macri, L. M., Hoffmann, S. L., et al. 2016, ApJ, 826, 56

Sterken, C. 2005, in The Light-Time Effect in Astrophysics: Causes and cures of the O-C diagram, ed. C. Sterken, ASP Conf. Ser., 335, 3

Storm, J., Carney, B. W., Gieren, W. P., et al. 2004, A\&A, 415, 531

Storm, J., Gieren, W., Fouqué, P., et al. 2011, A\&A, 534, A94

Turner, D. G., Kovtyukh, V. V., Luck, R. E., \& Berdnikov, L. N. 2013, ApJ, 772, L10

Voûte, R. 1939, Annals of the Bosscha Observatory Lembang (Java) Indonesia, 8,31

Walraven, T., Muller, A. B., \& Oosterhoff, P. T. 1958, Bull. Astron. Inst. Netherlands, 14, 81

Welch, D. L., Wieland, F., McAlary, C. W., et al. 1984, ApJS, 54, 547

Westerlund, B. E. 1963, MNRAS, 127, 71

Winters, J. G., Henry, T. J., Jao, W.-C., et al. 2011, AJ, 141, 21

Young, J. 2003, in EAS 6, EAS Publications Series, eds. G. Perrin, \& F. Malbet, 181 


\section{Appendix A: Photometric measurements}

Table A.1. O-C residuals for RS Puppis.

\begin{tabular}{|c|c|c|c|c|}
\hline $\begin{array}{c}\mathrm{JD}_{\odot} \\
-2.4 \times 10^{6}\end{array}$ & $E$ & $\begin{array}{r}\mathrm{O}-\mathrm{C} \\
{[\mathrm{d}]}\end{array}$ & $W$ & Reference \\
\hline 15050.6 & -977 & 85.1 & 1 & Innes \& Gill (1903) \\
\hline 15424.6 & -968 & 85.7 & 1 & Gerasimovic (1927) \\
\hline 16830.1 & -934 & 80.5 & 1 & Gerasimovic (1927) \\
\hline 18277.9 & -899 & 76.2 & 1 & Gerasimovic (1927) \\
\hline 19723.4 & -864 & 69.5 & 1 & Gerasimovic (1927) \\
\hline 20963.2 & -834 & 64.6 & 1 & Gerasimovic (1927) \\
\hline 22824.1 & -789 & 58.5 & 1 & Gerasimovic (1927) \\
\hline 26006.61 & -712 & 46.24 & 1 & Voûte (1939) \\
\hline 26379.42 & -703 & 45.64 & 1 & Voûte (1939) \\
\hline 26752.55 & -694 & 45.36 & 1 & Voûte (1939) \\
\hline 27042.15 & -687 & 44.53 & 1 & Voûte (1939) \\
\hline 27456.32 & -677 & 43.80 & 1 & Voûte (1939) \\
\hline 27828.94 & -668 & 43.01 & 1 & Voûte (1939) \\
\hline 28201.64 & -659 & 42.30 & 1 & Voûte (1939) \\
\hline 34451.700 & -508 & 27.366 & 1 & Eggen et al. (1957) \\
\hline 34533.607 & -506 & 26.293 & 2 & Walraven et al. (1958) \\
\hline 34864.914 & -498 & 25.680 & 2 & Eggen et al. (1957) \\
\hline 35196.511 & -490 & 25.357 & 1 & Irwin (1961) \\
\hline 37513.865 & -434 & 19.271 & 3 & Westerlund (1963) \\
\hline 37638.297 & -431 & 19.233 & 3 & Mitchell et al. (1964) \\
\hline 40701.394 & -357 & 12.070 & 3 & Pel (1976) \\
\hline 40949.305 & -351 & 11.041 & 3 & Pel (1976) \\
\hline 41405.313 & -340 & 10.659 & 1 & Dean et al. (1977) \\
\hline 41735.214 & -332 & 8.640 & 2 & Madore (1975) \\
\hline 41776.643 & -331 & 8.579 & 3 & Dean et al. (1977) \\
\hline 42065.736 & -324 & 7.242 & 3 & Dean et al. (1977) \\
\hline 42438.472 & -315 & 6.568 & 2 & Dean et al. (1977) \\
\hline 44055.085 & -276 & 5.071 & 2 & Harris (1980) \\
\hline 44428.140 & -267 & 4.716 & 1 & Moffett \& Barnes (1984) \\
\hline 44967.089 & -254 & 4.295 & 3 & Moffett \& Barnes (1984) \\
\hline 47951.011 & -182 & 0.937 & 3 & ESA (1997) \\
\hline 48240.973 & -175 & 0.469 & 3 & ESA (1997) \\
\hline 48448.945 & -170 & 0.991 & 3 & ESA (1997) \\
\hline 48739.114 & -163 & 0.730 & 3 & ESA (1997) \\
\hline 49111.661 & -154 & -0.133 & 1 & AAVSO \\
\hline 49360.599 & -148 & -0.135 & 1 & Walker \& Williams* \\
\hline 49527.027 & -144 & 0.333 & 1 & AAVSO \\
\hline 49817.467 & -137 & 0.343 & 3 & Berdnikov $(1995,2008)$ \\
\hline 50108.216 & -130 & 0.662 & 3 & Bersier (2002) \\
\hline 50357.203 & -124 & 0.709 & 3 & Berdnikov $(1995,2008)$ \\
\hline 50522.961 & -120 & 0.507 & 3 & Bersier (2002) \\
\hline 50564.680 & -119 & 0.736 & 1 & Berdnikov $(1995,2008)$ \\
\hline 51270.917 & -102 & 1.643 & 1 & Berdnikov $(1995,2008)$ \\
\hline 51644.316 & -93 & 1.632 & 2 & Berdnikov $(1995,2008)$ \\
\hline 51976.659 & -85 & 2.055 & 3 & Berdnikov $(1995,2008)$ \\
\hline 52350.347 & -76 & 2.333 & 3 & Berdnikov $(1995,2008)$ \\
\hline 52640.475 & -69 & 2.031 & 3 & Berdnikov $(1995,2008)$ \\
\hline 52681.984 & -68 & 2.050 & 3 & Pojmanski (2002) \\
\hline 53014.205 & -60 & 2.351 & 2 & Berdnikov $(1995,2008)$ \\
\hline 53055.383 & -59 & 2.039 & 3 & Pojmanski (2002) \\
\hline 53096.193 & -58 & 1.359 & 3 & Berdnikov $(1995,2008)$ \\
\hline
\end{tabular}

Notes. $\mathrm{JD}_{\odot}$ is the heliocentric moment of maximum brightness. $E$ is the epoch number as calculated from the ephemeris $C=2455501.254+$ $41.49 \times E$. $W$ is the weight assigned to the $\mathrm{O}-\mathrm{C}$ value $(1,2$, or 3 depending on the quality of the light curve). The ${ }^{*}$ symbol in the references indicates unpublished data.
Table A.1. continued.

\begin{tabular}{crrll}
\hline \hline $\begin{array}{c}\mathrm{JD}_{\odot} \\
-2.4 \times 10^{6}\end{array}$ & $E$ & $\begin{array}{r}\mathrm{O}-\mathrm{C} \\
{[\mathrm{d}]}\end{array}$ & $W$ & Reference \\
\hline 53551.918 & -47 & 0.694 & 2 & Tabur* \\
53842.397 & -40 & 0.743 & 2 & AAVSO \\
53842.461 & -40 & 0.807 & 3 & Tabur* \\
53842.495 & -40 & 0.841 & 2 & Pojmanski (2002) \\
54091.419 & -34 & 0.825 & 3 & present work \\
54132.197 & -33 & 0.113 & 3 & AAVSO \\
54215.229 & -31 & 0.165 & 3 & Tabur* \\
54215.264 & -31 & 0.200 & 3 & Pojmanski (2002) \\
54505.241 & -24 & -0.253 & 3 & Pojmanski (2002) \\
54546.574 & -23 & -0.410 & 3 & Tabur* \\
54546.611 & -23 & -0.373 & 3 & AAVSO \\
54588.094 & -22 & -0.380 & 2 & present work \\
54837.503 & -16 & 0.089 & 3 & Pojmanski (2002) \\
54878.846 & -15 & -0.058 & 2 & AAVSO \\
55127.522 & -9 & -0.322 & 1 & Pojmanski (2002) \\
55252.067 & -6 & -0.247 & 2 & AAVSO \\
55252.640 & -6 & -0.241 & 3 & Kervella et al. (2014) \\
55293.713 & -5 & -0.091 & 2 & present work \\
55335.074 & -4 & -0.220 & 3 & present work \\
55501.254 & 0 & 0.000 & 3 & present work \\
55625.868 & 3 & 0.144 & 2 & AAVSO \\
56041.317 & 13 & 0.693 & 3 & AAVSO \\
56373.179 & 21 & 0.635 & 3 & AAVSO \\
\hline
\end{tabular}


P. Kervella et al.: Projection factor of the long-period Cepheid RS Pup

Table A.2. SMARTS photometry of RS Pup.

\begin{tabular}{|c|c|c|c|c|}
\hline $\mathrm{JD}_{\odot}-2.4 \times 10^{6}$ & Phase & $B$ & $V$ & $R$ \\
\hline 54525.7016 & 0.0000 & 8.907 & 7.217 & - \\
\hline 54532.6658 & 0.1678 & 9.211 & 7.559 & 6.690 \\
\hline 54536.6723 & 0.2643 & 9.389 & 7.647 & 6.823 \\
\hline 54539.6256 & 0.3354 & 9.257 & - & - \\
\hline 54543.7118 & 0.4339 & 8.094 & - & - \\
\hline 54558.5980 & 0.7925 & 8.508 & - & - \\
\hline 54563.5447 & 0.9116 & 8.732 & - & - \\
\hline 54567.6262 & 0.0100 & 8.936 & 7.255 & - \\
\hline 54569.5540 & 0.0564 & 9.061 & 7.304 & - \\
\hline 54576.6180 & 0.2266 & 9.370 & 7.632 & - \\
\hline 54578.5235 & 0.2725 & 9.418 & 7.657 & - \\
\hline 54585.5011 & 0.4406 & 7.999 & - & - \\
\hline 54587.5767 & 0.4906 & 7.717 & 6.565 & - \\
\hline 54592.5966 & 0.6115 & 7.979 & - & - \\
\hline 54596.4998 & 0.7055 & 8.280 & - & - \\
\hline 54606.4899 & 0.9462 & 8.778 & - & - \\
\hline 54608.4538 & 0.9935 & 8.897 & - & - \\
\hline 54610.5160 & 0.0432 & 8.999 & 7.319 & 6.476 \\
\hline 54616.5077 & 0.1875 & 9.226 & 7.626 & 6.684 \\
\hline 54623.4738 & 0.3553 & 9.046 & 7.502 & 6.745 \\
\hline 54628.4718 & 0.4757 & 7.759 & 6.562 & - \\
\hline 54635.4700 & 0.6443 & 8.021 & 6.743 & - \\
\hline 54643.4491 & 0.8365 & 8.651 & 7.045 & - \\
\hline 54725.8512 & 0.8216 & 8.581 & - & - \\
\hline 54729.8645 & 0.9182 & 8.728 & - & - \\
\hline 54733.8631 & 0.0146 & 8.901 & - & - \\
\hline 54737.8615 & 0.1109 & 9.158 & - & - \\
\hline 54741.8534 & 0.2071 & 9.369 & - & - \\
\hline 54745.8538 & 0.3034 & 9.358 & - & - \\
\hline 54749.8616 & 0.4000 & 8.516 & - & - \\
\hline 54753.8324 & 0.4956 & 7.697 & - & - \\
\hline 54757.8274 & 0.5919 & 7.943 & - & - \\
\hline 54761.7860 & 0.6872 & 8.230 & - & - \\
\hline 54765.8078 & 0.7841 & 8.504 & - & - \\
\hline 54769.8674 & 0.8819 & 8.714 & - & - \\
\hline 54773.8301 & 0.9774 & 8.843 & - & - \\
\hline 54781.8260 & 0.1700 & 9.290 & - & - \\
\hline 54786.7894 & 0.2896 & 9.420 & - & - \\
\hline 54790.7745 & 0.3856 & 8.677 & - & - \\
\hline 54794.8251 & 0.4831 & 7.737 & - & - \\
\hline 54798.7928 & 0.5787 & 7.889 & - & - \\
\hline 54802.7976 & 0.6752 & 8.192 & - & - \\
\hline 54806.7729 & 0.7710 & 8.446 & - & - \\
\hline 54810.7922 & 0.8678 & 8.634 & - & - \\
\hline 54814.8080 & 0.9645 & 8.848 & - & - \\
\hline 54818.7459 & 0.0594 & 9.037 & - & - \\
\hline 54822.7881 & 0.1568 & 9.281 & - & - \\
\hline 54826.8193 & 0.2539 & 9.434 & - & - \\
\hline 54834.8558 & 0.4475 & 7.930 & - & - \\
\hline 54838.8136 & 0.5428 & 7.805 & - & - \\
\hline 54842.7421 & 0.6375 & 8.076 & - & - \\
\hline 54850.6641 & 0.8283 & 8.558 & - & - \\
\hline 54854.7633 & 0.9270 & 8.777 & - & - \\
\hline 54858.8023 & 0.0243 & 8.933 & - & - \\
\hline 54862.7753 & 0.1200 & 9.191 & - & - \\
\hline 54870.7043 & 0.3111 & 9.336 & - & - \\
\hline 54874.6945 & 0.4072 & 8.426 & - & - \\
\hline
\end{tabular}

Notes. The phases were computed assuming $P=41.5113$ days and the maximum light epoch $\mathrm{JD}_{\odot}=2455501.254$.
Table A.2. continued.

\begin{tabular}{|c|c|c|c|c|}
\hline $\mathrm{JD}_{\odot}-2.4 \times 10^{6}$ & Phase & $B$ & V & $R$ \\
\hline 54878.6485 & 0.5024 & 7.742 & - & - \\
\hline 54882.7127 & 0.6003 & 7.937 & - & - \\
\hline 54887.6628 & 0.7196 & 8.272 & - & - \\
\hline 54891.7283 & 0.8175 & 8.557 & - & - \\
\hline 54893.7053 & 0.8651 & 8.636 & - & - \\
\hline 54894.6760 & 0.8885 & 8.686 & - & - \\
\hline 54898.6346 & 0.9839 & 8.878 & - & - \\
\hline 54900.6782 & 0.0331 & 8.981 & - & - \\
\hline 54905.6835 & 0.1537 & 9.239 & - & - \\
\hline 54909.6473 & 0.2492 & 9.388 & - & - \\
\hline 54913.5922 & 0.3442 & 9.119 & - & - \\
\hline 54918.6438 & 0.4659 & 7.795 & - & - \\
\hline 54922.6326 & 0.5620 & 7.823 & - & - \\
\hline 54927.5957 & 0.6816 & 8.212 & - & - \\
\hline 54931.6133 & 0.7783 & 8.455 & - & - \\
\hline 54934.5792 & 0.8498 & 8.600 & - & - \\
\hline 54938.6068 & 0.9468 & 8.796 & - & - \\
\hline 54941.6177 & 0.0193 & 8.945 & - & - \\
\hline 54947.5678 & 0.1627 & 9.280 & - & - \\
\hline 54951.5816 & 0.2594 & 9.358 & - & - \\
\hline 54955.5251 & 0.3544 & 9.042 & - & - \\
\hline 54959.5129 & 0.4504 & 7.861 & - & - \\
\hline 54963.5287 & 0.5472 & 7.814 & - & - \\
\hline 54965.5290 & 0.5954 & 7.972 & - & - \\
\hline 54967.5193 & 0.6433 & 8.090 & - & - \\
\hline 54971.5113 & 0.7395 & 8.331 & - & - \\
\hline 54973.4828 & 0.7870 & 8.458 & - & - \\
\hline 54978.4828 & 0.9074 & 8.727 & - & - \\
\hline 54983.4894 & 0.0280 & 8.963 & - & - \\
\hline 54987.4693 & 0.1239 & 9.120 & - & - \\
\hline 54991.4909 & 0.2208 & 9.351 & - & - \\
\hline 54995.4676 & 0.3166 & 9.296 & - & - \\
\hline 55085.8831 & 0.4947 & 7.701 & - & - \\
\hline 55089.8848 & 0.5911 & 7.910 & - & - \\
\hline 55093.8648 & 0.6870 & 8.218 & - & - \\
\hline 55097.8738 & 0.7835 & 8.489 & - & - \\
\hline 55103.8741 & 0.9281 & 8.738 & - & - \\
\hline 55109.8788 & 0.0727 & 9.079 & - & - \\
\hline 55113.8235 & 0.1678 & 9.256 & - & - \\
\hline 55120.8393 & 0.3368 & 9.185 & - & - \\
\hline 55123.8805 & 0.4100 & 8.325 & - & - \\
\hline 55127.7931 & 0.5043 & 7.741 & - & - \\
\hline 55134.8193 & 0.6735 & 8.204 & - & - \\
\hline 55138.8452 & 0.7705 & 8.443 & - & - \\
\hline 55143.7863 & 0.8896 & 8.687 & - & - \\
\hline 55147.8228 & 0.9868 & 8.885 & - & - \\
\hline 55151.8117 & 0.0829 & 9.079 & - & - \\
\hline 55155.8085 & 0.1792 & 9.292 & - & - \\
\hline 55159.8390 & 0.2763 & 9.385 & - & - \\
\hline 55163.8296 & 0.3724 & 8.785 & - & - \\
\hline 55164.8319 & 0.3965 & 8.480 & - & - \\
\hline 55177.7902 & 0.7087 & 8.281 & - & - \\
\hline 55186.7513 & 0.9246 & 8.753 & - & - \\
\hline 55190.7915 & 0.0219 & 8.945 & - & - \\
\hline 55194.8218 & 0.1190 & 9.168 & - & - \\
\hline 55199.7529 & 0.2378 & 9.412 & - & - \\
\hline 55203.7830 & 0.3349 & 9.176 & - & - \\
\hline 55208.7977 & 0.4557 & 7.843 & - & - \\
\hline 55212.7897 & 0.5518 & 7.806 & - & 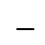 \\
\hline
\end{tabular}


Table A.2. continued.

\begin{tabular}{|c|c|c|c|c|}
\hline $\mathrm{JD}_{\odot}-2.4 \times 10^{6}$ & Phase & $B$ & V & $R$ \\
\hline 55216.7254 & 0.6466 & 8.109 & - & - \\
\hline 55220.7906 & 0.7446 & 8.355 & - & - \\
\hline 55224.7630 & 0.8403 & 8.580 & - & - \\
\hline 55228.7727 & 0.9369 & 8.794 & - & - \\
\hline 55232.7692 & 0.0331 & 9.043 & - & - \\
\hline 55235.7519 & 0.1050 & 9.163 & - & - \\
\hline 55240.7350 & 0.2250 & 9.394 & - & - \\
\hline 55244.7295 & 0.3213 & 9.301 & - & - \\
\hline 55248.6259 & 0.4151 & 8.282 & - & - \\
\hline 55252.6934 & 0.5131 & 7.726 & - & - \\
\hline 55258.6595 & 0.6568 & 8.129 & - & - \\
\hline 55261.6721 & 0.7294 & 8.342 & - & - \\
\hline 55261.7068 & 0.7302 & 8.328 & - & - \\
\hline 55263.7155 & 0.7786 & 8.457 & 6.943 & - \\
\hline 55263.7161 & 0.7786 & 8.469 & 6.928 & - \\
\hline 55267.6559 & 0.8736 & 8.673 & - & - \\
\hline 55267.6564 & 0.8736 & 8.661 & - & - \\
\hline 55271.6236 & 0.9691 & 8.874 & - & - \\
\hline 55271.6242 & 0.9691 & 8.877 & - & - \\
\hline 55279.7040 & 0.1638 & 9.243 & 7.511 & 6.642 \\
\hline 55279.7045 & 0.1638 & 9.259 & 7.523 & 6.651 \\
\hline 55283.5912 & 0.2574 & 9.416 & 7.689 & - \\
\hline 55283.5919 & 0.2574 & 9.424 & 7.666 & - \\
\hline 55286.6265 & 0.3305 & 9.243 & 7.589 & - \\
\hline 55286.6272 & 0.3306 & 9.224 & 7.586 & - \\
\hline 55290.6291 & 0.4270 & 8.126 & 6.857 & - \\
\hline 55290.6298 & 0.4270 & 8.131 & 6.871 & - \\
\hline 55293.5741 & 0.4979 & 7.724 & - & - \\
\hline 55293.5747 & 0.4979 & 7.716 & - & - \\
\hline 55299.5718 & 0.6424 & 8.082 & - & - \\
\hline 55299.5724 & 0.6424 & 8.087 & - & - \\
\hline 55303.5526 & 0.7383 & 8.367 & 6.850 & 6.151 \\
\hline 55303.5532 & 0.7383 & 8.338 & 6.889 & 6.138 \\
\hline 55307.5114 & 0.8337 & 8.581 & - & - \\
\hline 55307.5120 & 0.8337 & 8.580 & - & - \\
\hline 55312.5013 & 0.9539 & 8.858 & 7.186 & - \\
\hline 55312.5020 & 0.9539 & 8.854 & 7.196 & - \\
\hline 55317.5405 & 0.0753 & 9.099 & 7.382 & 6.550 \\
\hline 55317.5410 & 0.0753 & 9.104 & 7.375 & 6.537 \\
\hline 55321.5120 & 0.1709 & 9.313 & 7.561 & 6.708 \\
\hline 55321.5126 & 0.1709 & 9.306 & 7.563 & - \\
\hline 55325.5007 & 0.2670 & 9.426 & 7.682 & - \\
\hline 55325.5014 & 0.2670 & 9.435 & 7.690 & - \\
\hline 55335.4743 & 0.5073 & 7.698 & - & - \\
\hline 55335.4749 & 0.5073 & 7.706 & - & - \\
\hline 55340.5009 & 0.6284 & 8.071 & 6.717 & 6.053 \\
\hline 55340.5015 & 0.6284 & 8.095 & 6.753 & 6.081 \\
\hline 55343.4582 & 0.6996 & 8.273 & - & - \\
\hline 55343.4587 & 0.6996 & 8.263 & - & - \\
\hline 55348.4601 & 0.8201 & 8.604 & 6.990 & - \\
\hline 55348.4608 & 0.8201 & 8.557 & 6.995 & - \\
\hline 55352.4589 & 0.9164 & 8.786 & 7.143 & - \\
\hline 55352.4595 & 0.9165 & 8.778 & 7.141 & - \\
\hline 55355.4868 & 0.9894 & 8.947 & 7.243 & - \\
\hline 55355.4874 & 0.9894 & 8.917 & 7.253 & - \\
\hline 55359.4794 & 0.0856 & 9.138 & 7.414 & 6.551 \\
\hline 55359.4799 & 0.0856 & 9.081 & 7.400 & 6.535 \\
\hline 55365.4575 & 0.2296 & 9.393 & 7.666 & 6.787 \\
\hline 55365.4581 & 0.2296 & 9.399 & 7.666 & 6.788 \\
\hline 55369.4463 & 0.3257 & 9.289 & 7.607 & 6.796 \\
\hline
\end{tabular}

Table A.2. continued.

\begin{tabular}{|c|c|c|c|c|}
\hline $\mathrm{JD}_{\odot}-2.4 \times 10^{6}$ & Phase & $B$ & $V$ & $R$ \\
\hline 55369.4469 & 0.3257 & 9.306 & 7.619 & 6.804 \\
\hline 55374.4683 & 0.4466 & 7.904 & 6.676 & 6.029 \\
\hline 55374.4689 & 0.4467 & 7.889 & 6.642 & 6.104 \\
\hline 55378.4333 & 0.5422 & 7.739 & - & - \\
\hline 55378.4339 & 0.5422 & 7.786 & - & - \\
\hline 55430.9213 & 0.8066 & 8.537 & 6.971 & 6.208 \\
\hline 55430.9219 & 0.8066 & 8.565 & 6.965 & 6.185 \\
\hline 55431.9315 & 0.8309 & 8.557 & 7.037 & 6.248 \\
\hline 55431.9320 & 0.8309 & 8.598 & 7.076 & 6.119 \\
\hline 55435.9023 & 0.9266 & 8.786 & 7.138 & - \\
\hline 55435.9029 & 0.9266 & 8.809 & 7.156 & - \\
\hline 55438.9121 & 0.9991 & 8.974 & 7.254 & - \\
\hline 55438.9127 & 0.9991 & 8.938 & 7.243 & - \\
\hline 55446.9056 & 0.1916 & 9.333 & 7.604 & 6.732 \\
\hline 55446.9062 & 0.1917 & 9.301 & 7.573 & - \\
\hline 55453.8738 & 0.3595 & 8.919 & 7.398 & 6.585 \\
\hline 55453.8743 & 0.3595 & 8.897 & 7.397 & 6.598 \\
\hline 55456.9115 & 0.4327 & 7.983 & 6.787 & - \\
\hline 55456.9123 & 0.4327 & 8.004 & 6.782 & - \\
\hline 55459.8894 & 0.5044 & 7.726 & 6.556 & 5.941 \\
\hline 55459.8900 & 0.5044 & 7.738 & 6.551 & - \\
\hline 55464.8508 & 0.6239 & 8.088 & 6.729 & - \\
\hline 55464.8515 & 0.6240 & 8.048 & 6.716 & - \\
\hline 55468.8700 & 0.7208 & 8.361 & 6.877 & - \\
\hline 55468.8707 & 0.7208 & 8.359 & 6.843 & - \\
\hline 55471.8227 & 0.7919 & 8.523 & 6.971 & - \\
\hline 55471.8234 & 0.7919 & 8.517 & 6.938 & - \\
\hline 55476.8630 & 0.9133 & 8.778 & - & - \\
\hline 55476.8636 & 0.9133 & 8.766 & - & - \\
\hline 55480.8274 & 0.0088 & 8.954 & 7.286 & 6.441 \\
\hline 55480.8280 & 0.0088 & 8.977 & 7.297 & - \\
\hline 55484.8926 & 0.1067 & 9.165 & 7.469 & 6.598 \\
\hline 55484.8931 & 0.1068 & 9.174 & 7.469 & 6.593 \\
\hline 55487.8774 & 0.1786 & 9.329 & 7.583 & - \\
\hline 55487.8780 & 0.1787 & 9.317 & 7.582 & - \\
\hline 55490.8463 & 0.2502 & 9.408 & 7.685 & - \\
\hline 55490.8469 & 0.2502 & 9.405 & 7.672 & - \\
\hline 55494.8314 & 0.3462 & 9.091 & 7.506 & 6.703 \\
\hline 55494.8320 & 0.3462 & 9.079 & 7.505 & 6.655 \\
\hline 55499.8628 & 0.4674 & 7.758 & 6.588 & - \\
\hline 55499.8634 & 0.4674 & 7.744 & - & - \\
\hline 55503.7804 & 0.5617 & 7.845 & - & - \\
\hline 55503.7809 & 0.5618 & 7.848 & - & - \\
\hline 55505.8172 & 0.6108 & 8.008 & - & - \\
\hline 55505.8178 & 0.6108 & 7.985 & - & - \\
\hline 55510.8182 & 0.7313 & 8.349 & - & - \\
\hline 55510.8188 & 0.7313 & 8.330 & - & - \\
\hline 55514.8232 & 0.8278 & 8.583 & - & - \\
\hline 55514.8238 & 0.8278 & 8.569 & - & - \\
\hline 55519.8138 & 0.9480 & 8.807 & 7.181 & - \\
\hline 55519.8145 & 0.9480 & 8.846 & 7.192 & - \\
\hline 55522.7774 & 0.0194 & 8.980 & 7.287 & - \\
\hline 55522.7780 & 0.0194 & 8.982 & 7.281 & - \\
\hline 55527.8711 & 0.1421 & 9.260 & 7.525 & - \\
\hline 55527.8718 & 0.1421 & 9.254 & 7.530 & - \\
\hline 55531.8225 & 0.2373 & 9.400 & 7.684 & 6.819 \\
\hline 55531.8231 & 0.2373 & 9.445 & 7.677 & - \\
\hline 55535.8033 & 0.3332 & 9.230 & 7.597 & 6.763 \\
\hline 55535.8039 & 0.3332 & 9.233 & 7.604 & - \\
\hline 55540.8641 & 0.4551 & 7.774 & - & - \\
\hline
\end{tabular}


P. Kervella et al.: Projection factor of the long-period Cepheid RS Pup

Table A.2. continued.

\begin{tabular}{ccccc}
\hline \hline $\mathrm{JD}_{\odot}-2.4 \times 10^{6}$ & Phase & $B$ & $V$ & $R$ \\
\hline 55540.8646 & 0.4551 & 7.801 & - & - \\
55541.7981 & 0.4776 & 7.698 & - & - \\
55541.7986 & 0.4776 & 7.700 & - & - \\
55542.8176 & 0.5021 & 7.687 & - & - \\
55542.8182 & 0.5022 & 7.663 & - & - \\
55543.7799 & 0.5253 & 7.718 & - & - \\
55543.7805 & 0.5253 & 7.731 & - & - \\
55545.8222 & 0.5745 & 7.867 & - & - \\
55545.8227 & 0.5745 & 7.873 & - & - \\
55546.8471 & 0.5992 & 7.930 & 6.624 & - \\
55546.8477 & 0.5992 & 7.945 & 6.653 & - \\
55547.8008 & 0.6222 & 8.034 & 6.690 & - \\
55547.8014 & 0.6222 & 8.017 & 6.683 & - \\
55548.7864 & 0.6459 & 8.085 & - & - \\
55548.7869 & 0.6459 & 8.091 & - & - \\
55549.8240 & 0.6709 & 8.165 & 6.821 & - \\
55549.8247 & 0.6709 & 8.142 & - & - \\
55550.7313 & 0.6928 & 8.233 & - & - \\
55550.7319 & 0.6928 & 8.191 & - & - \\
55551.7763 & 0.7180 & 8.278 & 6.838 & - \\
55551.7769 & 0.7180 & 8.300 & - & - \\
55577.7883 & 0.3446 & 9.122 & 7.526 & - \\
55577.7890 & 0.3446 & 9.137 & 7.520 & - \\
55578.7657 & 0.3681 & 8.861 & 7.368 & - \\
55578.7664 & 0.3681 & 8.865 & 7.362 & - \\
55579.7229 & 0.3912 & 8.586 & 7.180 & - \\
55579.7236 & 0.3912 & 8.564 & - & - \\
55581.7737 & 0.4406 & 7.940 & - & - \\
55581.7743 & 0.4406 & 7.925 & - & - \\
55582.7597 & 0.4643 & 7.762 & - & - \\
55582.7602 & 0.4644 & 7.757 & - & - \\
55583.7457 & 0.4881 & 7.705 & - & - \\
55583.7462 & 0.4881 & 7.680 & - & - \\
55584.7586 & 0.5125 & 7.703 & 6.563 & - \\
55584.7593 & 0.5125 & 7.706 & 6.541 & - \\
55585.7983 & 0.5375 & 7.776 & 6.573 & - \\
55585.7990 & 0.5376 & 7.797 & 6.579 & - \\
55586.7503 & 0.5605 & 7.818 & - & - \\
55586.7509 & 0.5605 & 7.818 & - & - \\
55587.7925 & 0.5856 & 7.904 & 6.642 & 5.993 \\
55587.7930 & 0.5856 & 7.906 & 6.648 & 6.000 \\
\hline & & & &
\end{tabular}

Table A.3. Photometry of RS Pup in the Johnson $V$ band collected by AAVSO observer Neil Butterworth (BIW) from Mt. Louisa, Australia using a transformed DSLR.

\begin{tabular}{|c|c|c|}
\hline $\mathrm{JD}_{\odot}-2.4 \times 10^{6}$ & Phase & V \\
\hline 56404.8860 & 0.7380 & $7.634 \pm 0.013$ \\
\hline 56406.8808 & 0.7861 & $7.608 \pm 0.017$ \\
\hline 56423.8730 & 0.1956 & $6.886 \pm 0.131$ \\
\hline 56424.8695 & 0.2196 & $6.854 \pm 0.008$ \\
\hline 56436.8738 & 0.5089 & $7.248 \pm 0.016$ \\
\hline 56455.8664 & 0.9666 & $6.556 \pm 0.012$ \\
\hline 56468.8603 & 0.2797 & $6.941 \pm 0.011$ \\
\hline 56471.8580 & 0.3519 & $7.050 \pm 0.020$ \\
\hline 56474.8600 & 0.4243 & $7.108 \pm 0.012$ \\
\hline 56702.9424 & 0.9256 & $6.799 \pm 0.026$ \\
\hline 56710.9415 & 0.1183 & $6.698 \pm 0.018$ \\
\hline 56731.9182 & 0.6238 & $7.457 \pm 0.024$ \\
\hline 56732.9219 & 0.6480 & $7.493 \pm 0.011$ \\
\hline 56733.9270 & 0.6722 & $7.552 \pm 0.014$ \\
\hline 56734.9298 & 0.6964 & $7.593 \pm 0.015$ \\
\hline 56749.9202 & 0.0576 & $6.612 \pm 0.019$ \\
\hline 56751.9200 & 0.1058 & $6.670 \pm 0.020$ \\
\hline 56754.9375 & 0.1785 & $6.772 \pm 0.017$ \\
\hline 56761.9075 & 0.3465 & $7.022 \pm 0.021$ \\
\hline 56764.8938 & 0.4184 & $7.110 \pm 0.025$ \\
\hline 56767.9004 & 0.4909 & $7.215 \pm 0.019$ \\
\hline 56775.8966 & 0.6836 & $7.583 \pm 0.017$ \\
\hline 56781.9189 & 0.8287 & $7.592 \pm 0.016$ \\
\hline 56784.8895 & 0.9003 & $7.047 \pm 0.019$ \\
\hline 56787.9128 & 0.9731 & $6.555 \pm 0.019$ \\
\hline 56798.9200 & 0.2384 & $6.850 \pm 0.025$ \\
\hline 56801.8710 & 0.3095 & $6.953 \pm 0.029$ \\
\hline 56819.8598 & 0.7430 & $7.633 \pm 0.037$ \\
\hline 56820.8664 & 0.7673 & $7.660 \pm 0.034$ \\
\hline 57095.9079 & 0.3948 & $7.053 \pm 0.009$ \\
\hline 57096.9064 & 0.4188 & $7.087 \pm 0.011$ \\
\hline 57099.9117 & 0.4912 & $7.189 \pm 0.008$ \\
\hline 57107.9034 & 0.6838 & $7.527 \pm 0.006$ \\
\hline 57108.9093 & 0.7081 & $7.563 \pm 0.006$ \\
\hline 57111.9094 & 0.7804 & $7.635 \pm 0.032$ \\
\hline 57136.8846 & 0.3822 & $7.048 \pm 0.028$ \\
\hline 57138.8867 & 0.4304 & $7.111 \pm 0.029$ \\
\hline 57139.8798 & 0.4543 & $7.153 \pm 0.033$ \\
\hline 57141.8801 & 0.5026 & $7.221 \pm 0.029$ \\
\hline 57142.8984 & 0.5271 & $7.258 \pm 0.032$ \\
\hline 57145.8928 & 0.5993 & $7.377 \pm 0.029$ \\
\hline 57147.8809 & 0.6472 & $7.477 \pm 0.030$ \\
\hline 57148.8779 & 0.6712 & $7.527 \pm 0.031$ \\
\hline 57150.8790 & 0.7194 & $7.589 \pm 0.028$ \\
\hline 57151.8755 & 0.7434 & $7.623 \pm 0.032$ \\
\hline 57153.8838 & 0.7918 & $7.638 \pm 0.031$ \\
\hline 57154.8876 & 0.8160 & $7.596 \pm 0.029$ \\
\hline 57161.8776 & 0.9844 & $6.528 \pm 0.033$ \\
\hline 57165.8708 & 0.0806 & $6.619 \pm 0.030$ \\
\hline 57198.8614 & 0.8757 & $7.236 \pm 0.033$ \\
\hline 57204.8609 & 0.0201 & $6.546 \pm 0.038$ \\
\hline 57392.9682 & 0.5517 & $7.278 \pm 0.016$ \\
\hline 57393.9915 & 0.5764 & $7.302 \pm 0.017$ \\
\hline 57394.9576 & 0.5996 & $7.339 \pm 0.017$ \\
\hline 57398.9627 & 0.6962 & $7.529 \pm 0.014$ \\
\hline 57399.9590 & 0.7202 & $7.567 \pm 0.025$ \\
\hline 57402.2453 & 0.7753 & $7.628 \pm 0.012$ \\
\hline
\end{tabular}


Table A.3. continued.

\begin{tabular}{ccc}
\hline \hline $\mathrm{JD}_{\odot}-2.4 \times 10^{6}$ & Phase & $V$ \\
\hline 57410.9215 & 0.9842 & $6.528 \pm 0.012$ \\
57433.9313 & 0.5388 & $7.231 \pm 0.019$ \\
57444.9741 & 0.8049 & $7.597 \pm 0.012$ \\
57446.8990 & 0.8513 & $7.411 \pm 0.015$ \\
57471.9630 & 0.4552 & $7.115 \pm 0.010$ \\
57477.9219 & 0.5988 & $7.351 \pm 0.009$ \\
57481.9689 & 0.6963 & $7.522 \pm 0.009$ \\
57490.9058 & 0.9117 & $6.920 \pm 0.009$ \\
57496.9034 & 0.0561 & $6.582 \pm 0.004$ \\
57509.9115 & 0.3696 & $7.014 \pm 0.012$ \\
57522.8794 & 0.6821 & $7.508 \pm 0.015$ \\
57523.8833 & 0.7063 & $7.557 \pm 0.008$ \\
57533.9424 & 0.9487 & $6.627 \pm 0.016$ \\
57536.8819 & 0.0194 & $6.532 \pm 0.009$ \\
57543.8699 & 0.1878 & $6.768 \pm 0.010$ \\
57544.8855 & 0.2123 & $6.803 \pm 0.012$ \\
57546.8602 & 0.2598 & $6.868 \pm 0.013$ \\
57547.8634 & 0.2840 & $6.889 \pm 0.014$ \\
\hline
\end{tabular}

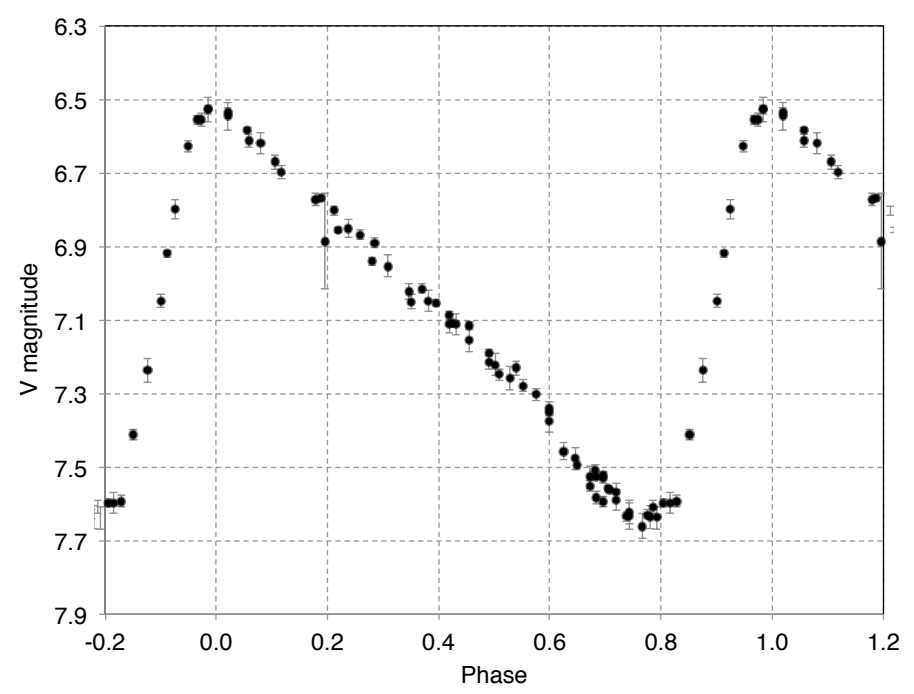

Fig. A.1. AAVSO light curve of RS Pup in the Johnson $V$ band.

\section{Appendix B: SPIPS analysis of separate pulsation cycles}

We present here the results of the SPIPS modeling of the four pulsation cycles of RS Pup observed by Anderson (2014). We keep in the dataset only the radial velocity data of one cycle, while keeping all the other datasets unchanged. The results are presented in Fig. B.1 to B.4. We also present in Figs. B.5 and B.6 the best-fit SPIPS solutions obtained considering separately the radial velocity datasets of Storm et al. (2004) and Anderson (2014), respectively. 
P. Kervella et al.: Projection factor of the long-period Cepheid RS Pup

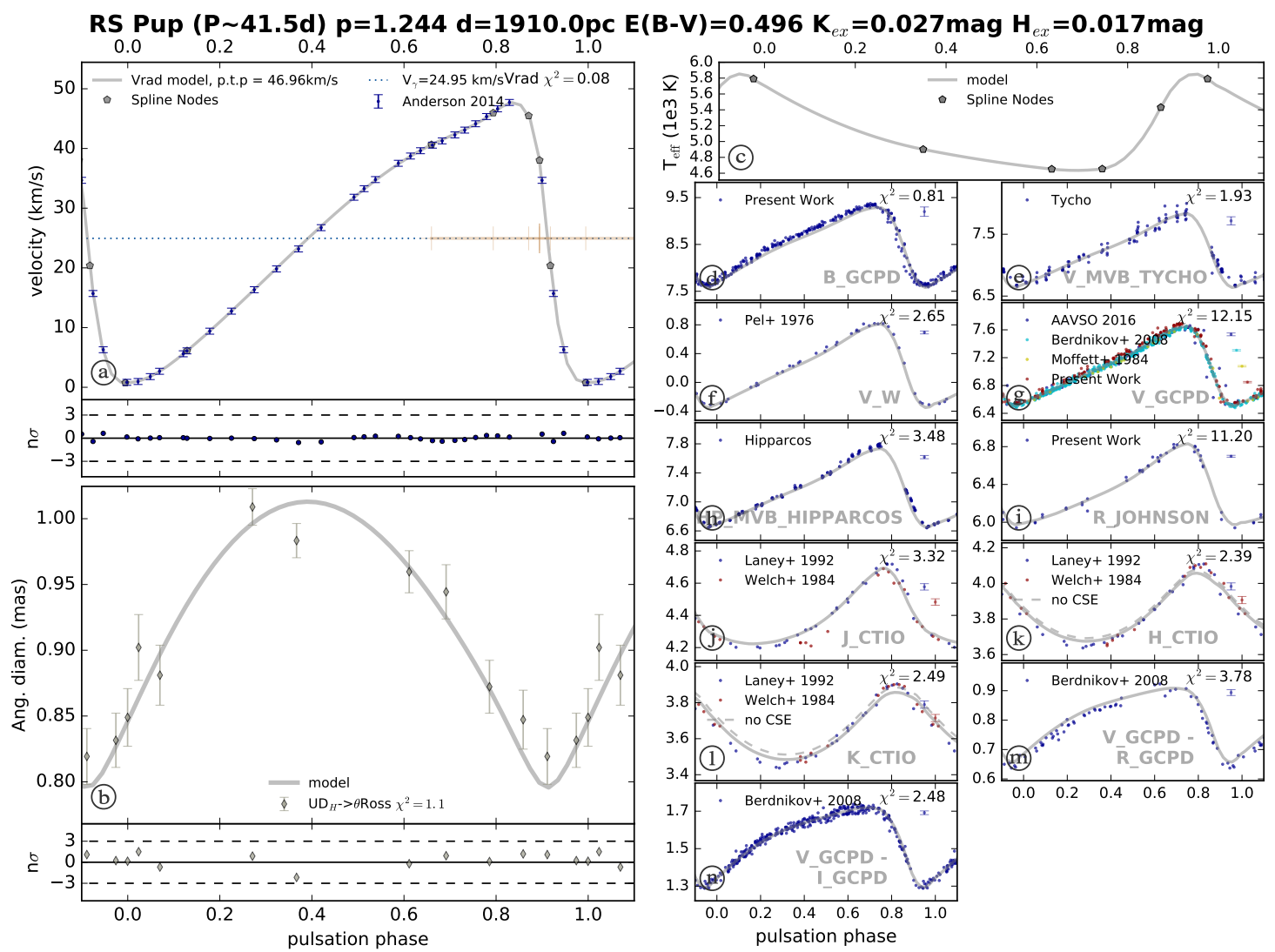

Fig. B.1. SPIPS model of RS Pup for the radial velocities of Cycle 1 of Anderson (2014).

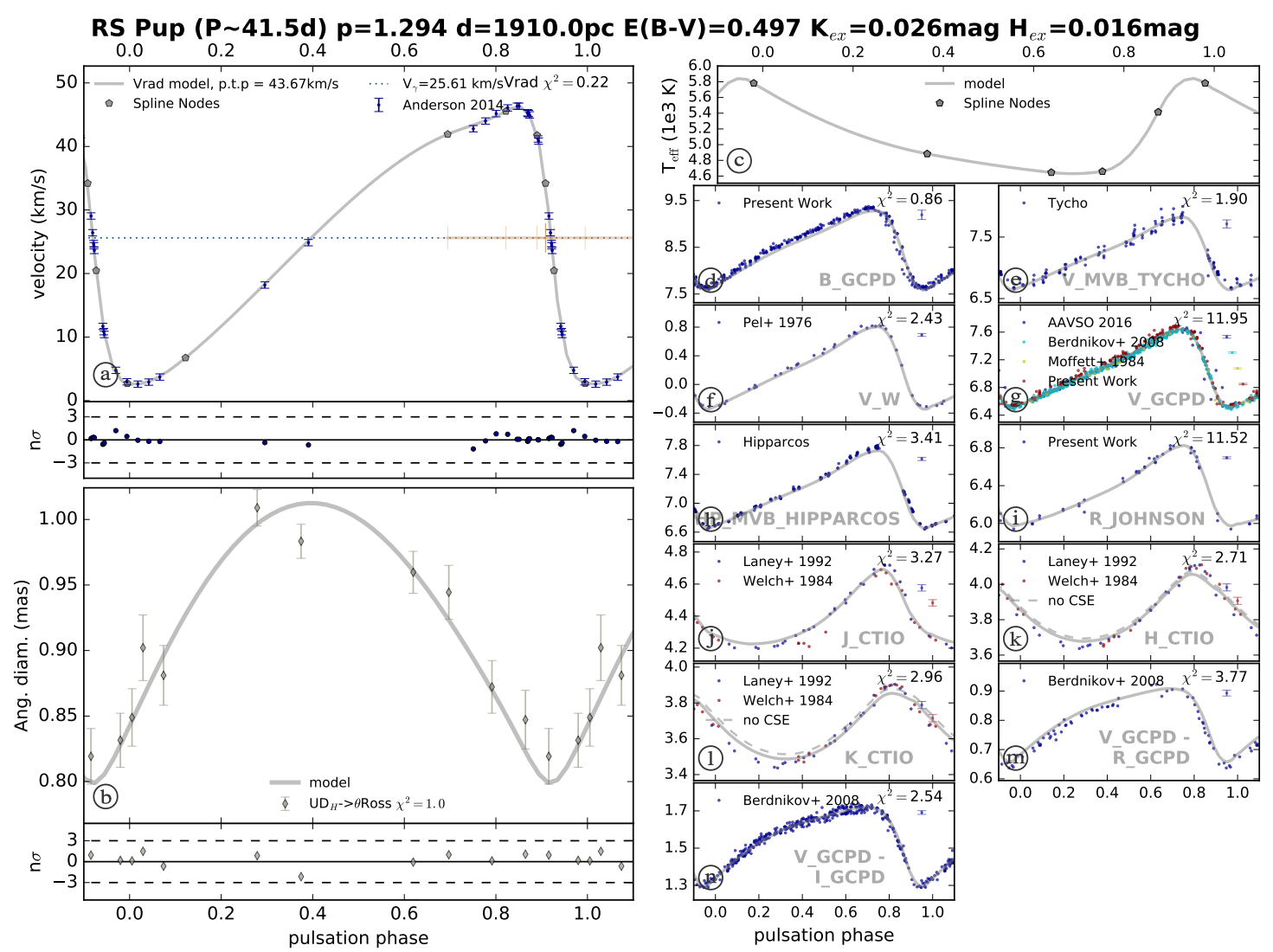

Fig. B.2. Same as Fig. B.1 for Cycle 2. 

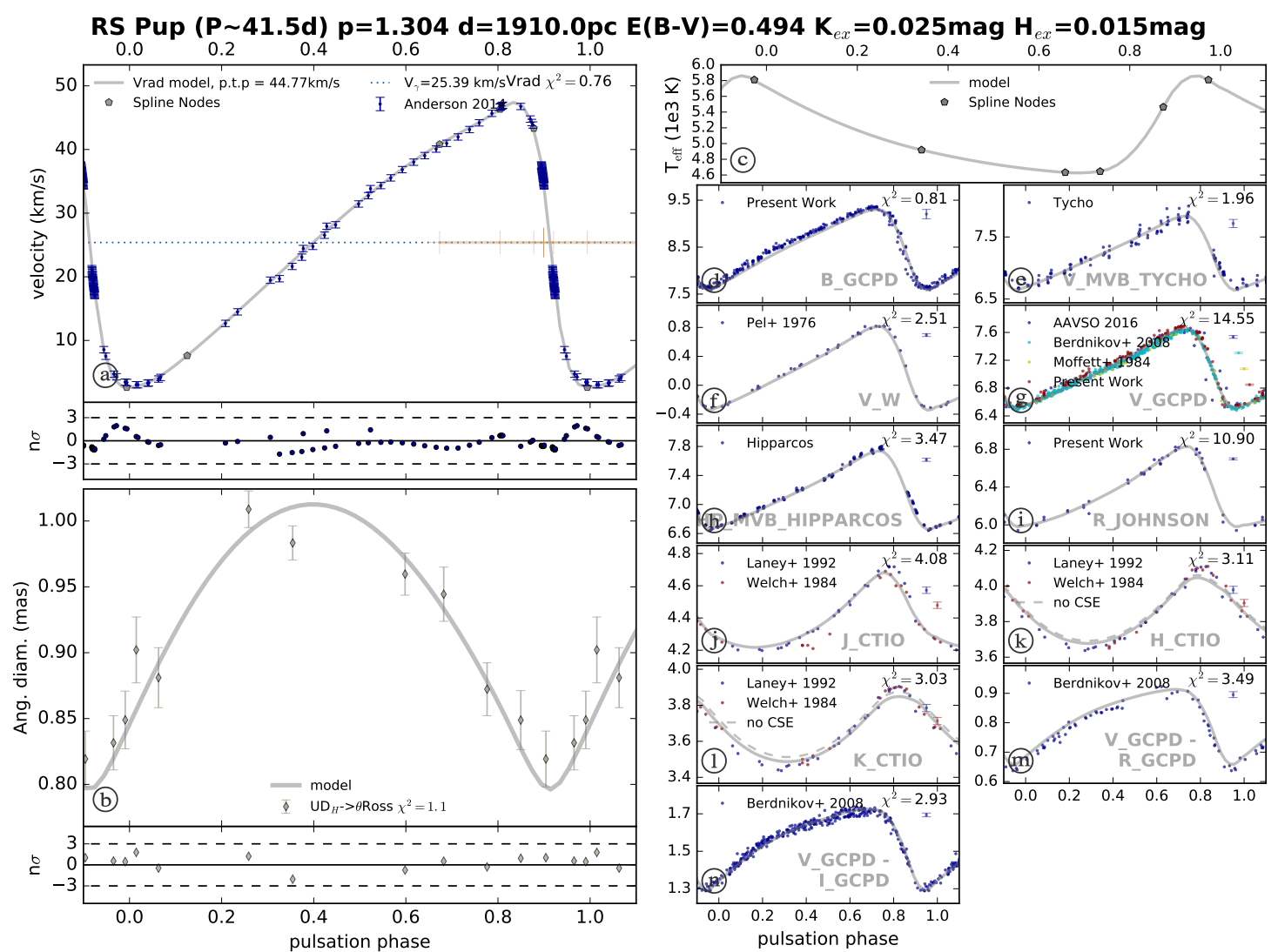

Fig. B.3. Same as Fig. B.1 for Cycle 3.
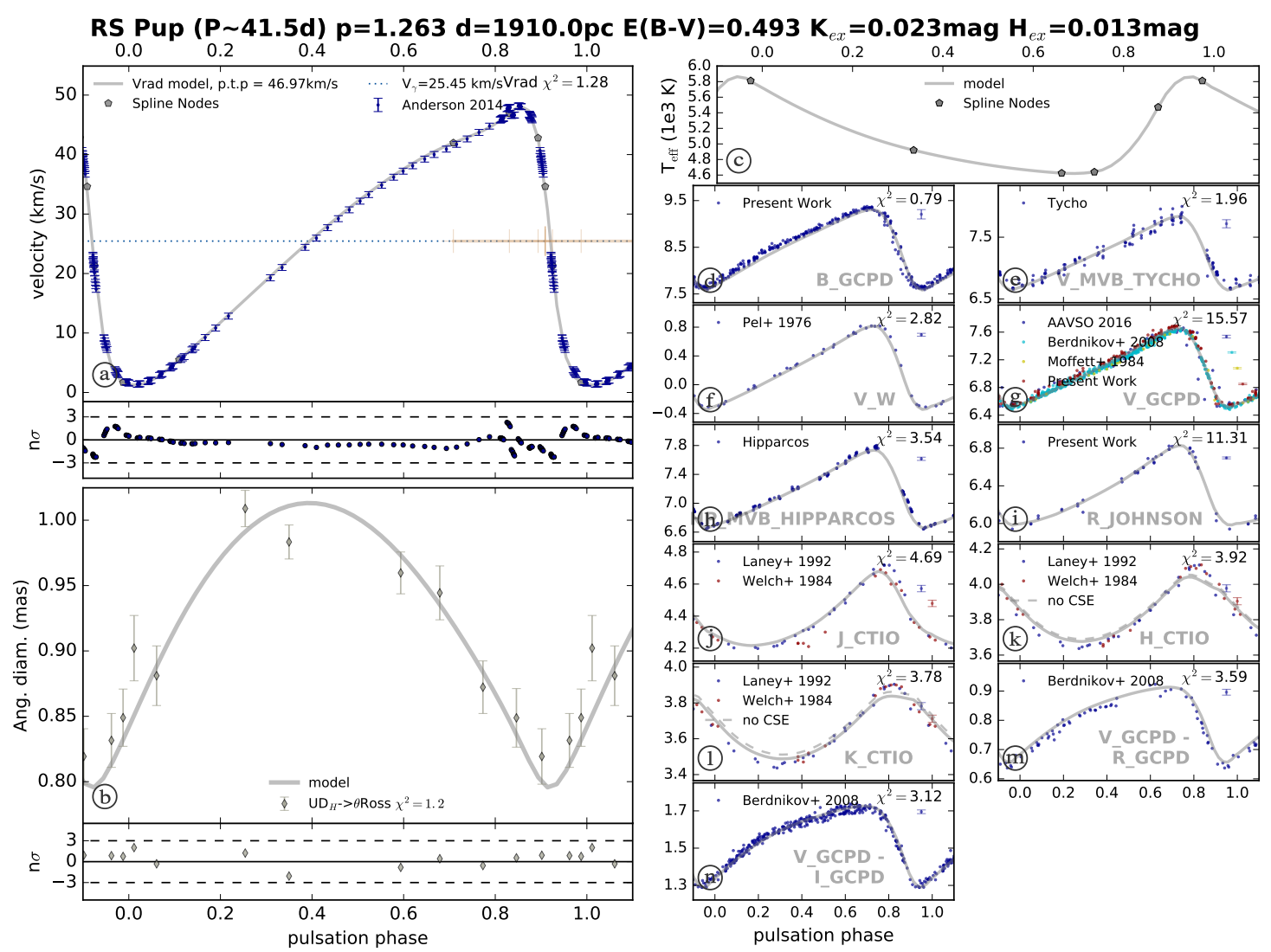

Fig. B.4. Same as Fig. B.1 for Cycle 4. 
P. Kervella et al.: Projection factor of the long-period Cepheid RS Pup

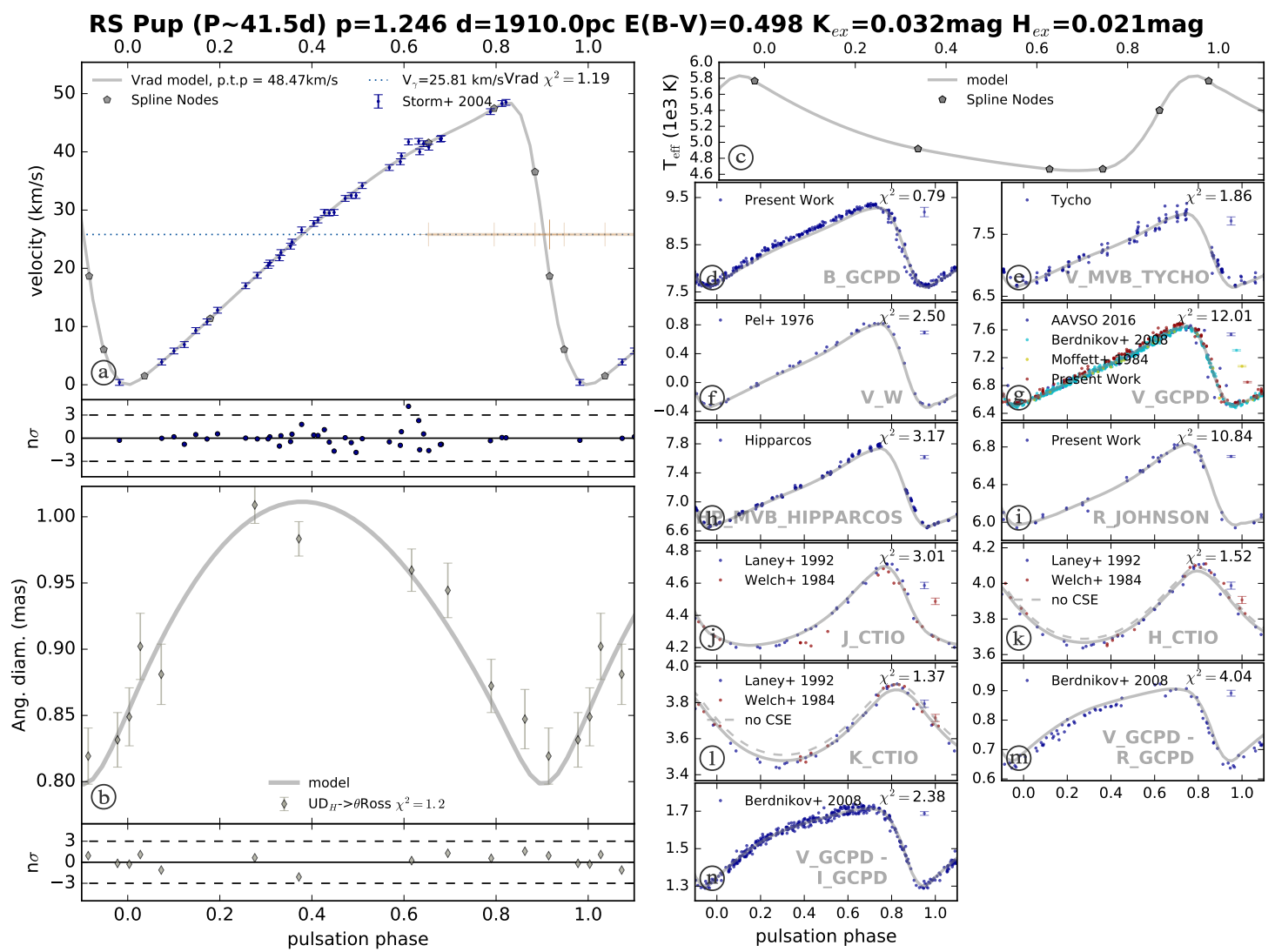

Fig. B.5. SPIPS model of RS Pup for the radial velocities collected exclusively by Storm et al. (2004).

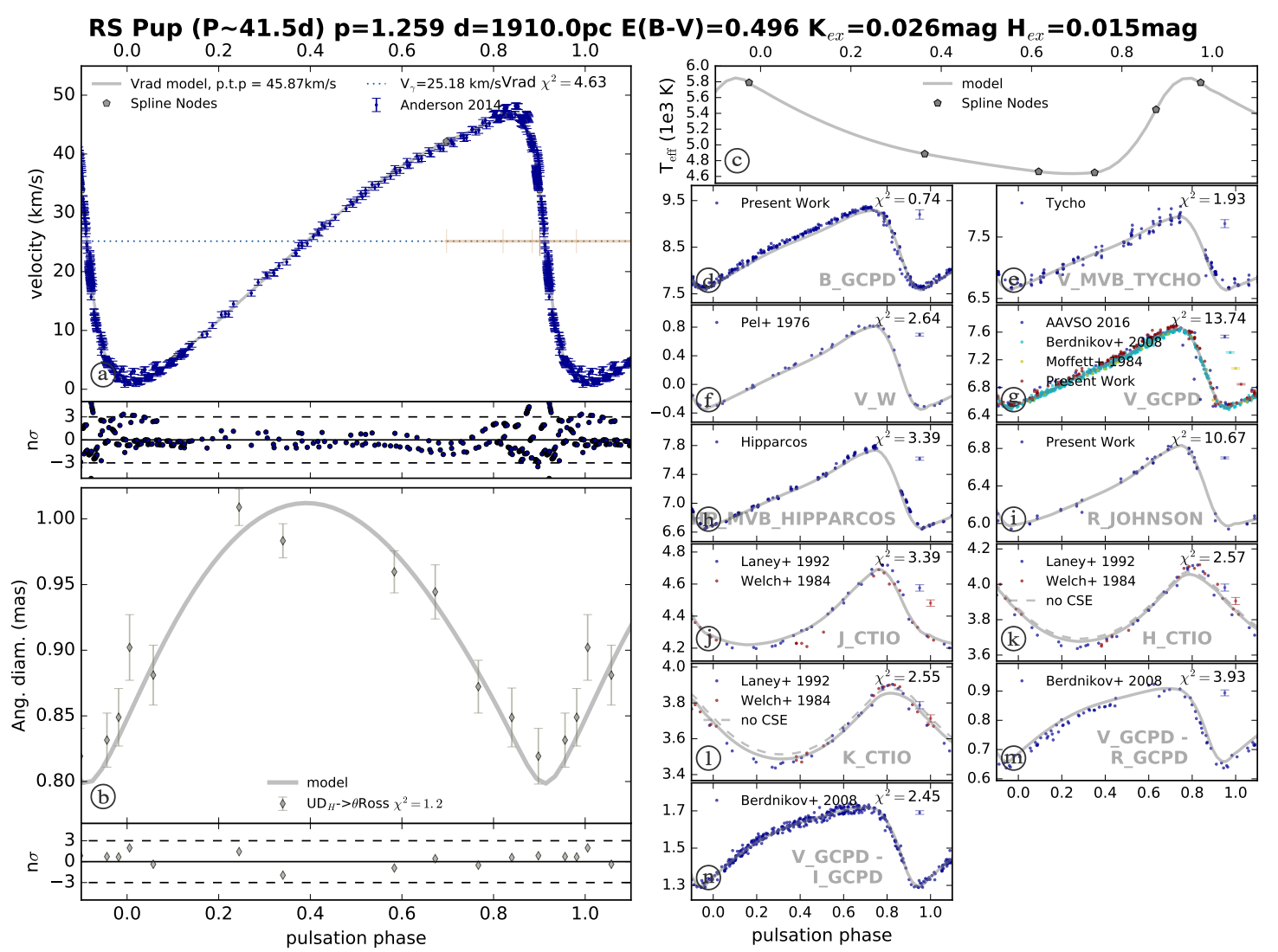

Fig. B.6. Same as Fig. B.5 for a combination of all cycles of the radial velocity observations by Anderson (2014). 